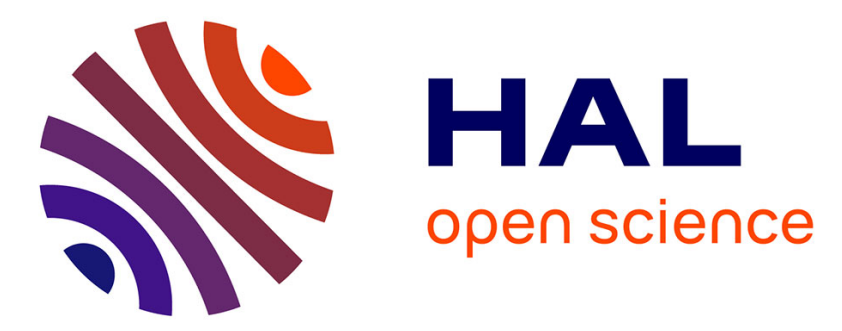

\title{
Solving inverse source problems using observability. Applications to the Euler-Bernoulli plate equation
}

Carlos Alves, Ana Leonor Silvestre, Takéo Takahashi, Marius Tucsnak

\section{To cite this version:}

Carlos Alves, Ana Leonor Silvestre, Takéo Takahashi, Marius Tucsnak. Solving inverse source problems using observability. Applications to the Euler-Bernoulli plate equation. SIAM Journal on Control and Optimization, 2009, 48 (3), pp.1632-1659. 10.1137/080725635 . hal-00283402

\section{HAL Id: hal-00283402 \\ https://hal.science/hal-00283402}

Submitted on 29 May 2008

HAL is a multi-disciplinary open access archive for the deposit and dissemination of scientific research documents, whether they are published or not. The documents may come from teaching and research institutions in France or abroad, or from public or private research centers.
L'archive ouverte pluridisciplinaire HAL, est destinée au dépôt et à la diffusion de documents scientifiques de niveau recherche, publiés ou non, émanant des établissements d'enseignement et de recherche français ou étrangers, des laboratoires publics ou privés. 


\title{
Solving inverse source problems using observability. Applications to the Euler-Bernoulli plate equation
}

\author{
Carlos Alves ${ }^{\dagger}$, Ana Leonor Silvestre ${ }^{\dagger}$, Takéo Takahashi*, Marius Tucsnak* \\ † Centro de Matemática e Aplicações and Department of Mathematics, \\ Instituto Superior Técnico (T.U. Lisbon), \\ Av. Rovisco Pais, 1049-001 Lisboa, Portugal \\ calves@math.ist.utl.pt, ana.silvestre@math.ist.utl.pt \\ * Institut Élie Cartan \\ UMR 7502, INRIA, Nancy-Université, CNRS, \\ POB239, 54506 Vandœuvre-lès-Nancy Cedex, France \\ takahash@iecn.u-nancy.fr, tucsnak@iecn.u-nancy.fr
}

\begin{abstract}
The aim of this paper is to provide a general framework allowing to use exact observability of infinite dimensional systems to solve a class of inverse source problems. More precisely, we show that if a system is exactly observable, then we can identify a source term in this system by knowing the corresponding intensity and appropriate observations which often correspond to the measure of some boundary traces. This abstract theory is then applied to a system governed by the EulerBernoulli plate equation. Using a different methodology, we show that exact observability can be used to identify both the locations and the intensities of combinations of point sources in the plate equation.
\end{abstract}

Keywords. inverse source problems, exact observability, plate equation

AMS subject classifications. 93C25; 93B07; 93C20; 35R30

\section{Introduction}

In this paper we consider the problem of determining sources in infinite dimensional systems by using appropriate observation operators. In the case of systems governed by PDE's these observation operators often correspond to the measure of some boundary traces. As it has been remarked in Puel and Yamamoto [18] this inverse problem is closely related to exact controllability properties (see also El Badia and Ha Duong in [8]).

One of our aims is to give a general framework, in terms of functional analysis, of the connection between exact observability (which is dual to exact controllability) and identifiability (possibly stable) of sources. In the case of sources of the form $\lambda(t) f$ with $\lambda:[0, \infty) \rightarrow \mathbb{C}$ given and $f$ unknown, this approach can be used to derive most of known results and some new ones, in particular for the Euler-Bernoulli plate equation.

In order to describe this abstract framework and our general results, let $X, Y$ be Hilbert spaces and $\mathcal{D}(A), \mathcal{D}(C)$ two subspaces of $X$. Let $A: \mathcal{D}(A) \rightarrow X$ be a generator of a strongly continuous group in $X$ and let $C: \mathcal{D}(C) \rightarrow Y$ be an observation operator. In this paper we study inverse source 
problems for the differential equation

$$
\begin{aligned}
\dot{z}(t) & =A z(t)+g(t) \quad(t \in(0, \tau)), \\
z(0) & =z_{0}, \\
y(t) & =C z(t) \quad(t \in(0, \tau)),
\end{aligned}
$$

where $\tau>0, z_{0} \in X$ are given and $g:[0, \tau] \rightarrow Z^{\prime}$ is (partially) unknown. Above $Z^{\prime} \supset X$ is the dual space of a space $Z \subset X$ containing $\mathcal{D}(A)$, as it will be made precise in Section 2. More precisely, we consider the problem of determining $g$ from appropriate measurements $y$ and the following classical questions associated to it:

- Identifiability: is the mapping $g \mapsto y$ one-to-one?

- Stability: assume that we have two sources $g^{(1)}$ and $g^{(2)}$ and let $y^{(1)}$ and $y^{(2)}$ be the corresponding observations. Can we find a positive constant $K$ such that

$$
\left\|g^{(1)}-g^{(2)}\right\| \leqslant K\left\|y^{(1)}-y^{(2)}\right\|
$$

with appropriate norms?

- Reconstruction: is it possible to "reconstruct", in some sense, $g$ from the observation $y$ ?

We will focus just on the first two topics and study them using a method that relies on the exact observability of the system

$$
\begin{gathered}
\dot{z}(t)=A z(t), \quad z(0)=z_{0}, \\
y(t)=C z(t) .
\end{gathered}
$$

Recall that system (1.4)-(1.5) is exactly observable in time $\tau>0$ if there exists $k_{\tau}>0$ such that

$$
\int_{0}^{\tau}\|y(t)\|_{Y}^{2} \mathrm{~d} t \geqslant k_{\tau}^{2}\left\|z_{0}\right\|_{X}^{2} \quad \forall z_{0} \in \mathcal{D}(A)
$$

One of our main results, Theorem 3.3, states that the exact observability in time $\tau>0$ of (1.4)(1.5) implies the stability (and identifiability) for the inverse source problem of (1.1)-(1.3) in the case where $g(t)=\lambda(t) f$, with $\lambda \in H^{1}(0, \tau)$ known, and $f \in Z^{\prime}$ to be determined.

As an example of application of this result, consider the following initial value problem for the Euler-Bernoulli plate equation

$$
\begin{cases}\frac{\partial^{2} w}{\partial t^{2}}+\Delta^{2} w=\lambda(t) \delta_{\xi} & \text { in }(0, \tau) \times \Omega, \\ w=\Delta w=0 & \text { on }(0, \tau) \times \partial \Omega, \\ w(0, x)=w_{0}(x), \frac{\partial w}{\partial t}(0, x)=w_{1}(x) & x \in \Omega,\end{cases}
$$

where $\Omega \subset \mathbb{R}^{2}$ is a bounded domain, $\tau>0, \lambda \in H^{1}(0, \tau)$ with $\lambda(0) \neq 0, \xi \in \Omega$ and $\delta_{\xi}$ is the Dirac mass concentrated in $\xi$. As a consequence of Theorem 3.3 below we will show that the mapping

$$
\Omega \rightarrow L^{2}\left(0, \tau ; L^{2}(\Gamma)\right), \quad \xi \mapsto y=\left.\frac{\partial w}{\partial \nu}\right|_{(0, \tau) \times \Gamma},
$$

where $\Gamma$ is a nonempty open subset of $\partial \Omega$, is well-defined and that if $\Omega$ is a rectangle $(0, a) \times(0, b)$ and $\Gamma$ contains both a horizontal and a vertical segment of nonzero length or if $\Omega$ is smooth and $\Gamma$ 
satisfies the geometric optics condition of Bardos, Lebeau, Rauch [3], then for all $\xi^{(1)}, \xi^{(2)} \in \Omega$, there exists $K>0$ such that

$$
\left|\xi^{(1)}-\xi^{(2)}\right| \leqslant K\left\|\frac{\partial w^{(1)}}{\partial \nu}-\frac{\partial w^{(2)}}{\partial \nu}\right\|_{L^{2}\left(0, \tau ; L^{2}(\Gamma)\right)} .
$$

In this inequality $w^{(1)}$ and $w^{(2)}$ are the solutions of (1.7) corresponding to $\xi^{(1)}$ and $\xi^{(2)}$, respectively, with suitable assumptions on the initial data.

We would like to emphasize that, since the only assumption on $A$ in (1.1)-(1.3) is that it generates a $C^{0}$ semigroup, our results can be applied, not only to the plate equations, but to a variety of problems including, for instance, the Schrödinger equation, the wave equation and the Maxwell system, by using appropriate exact observability results.

Another application of exact observability of the plates equation is shown in Theorem 5.2 which establishes identifiability for the inverse source problem

$$
\begin{cases}\frac{\partial^{2} w}{\partial t^{2}}+\Delta^{2} w=\sum_{j=1}^{N} \lambda_{j}(t) \delta_{\xi_{j}} & \text { in }(0, \tau) \times \Omega \\ w=\Delta w=0 & \text { on }(0, \tau) \times \partial \Omega \\ w(0, x)=w_{0}(x), \frac{\partial w}{\partial t}(0, x)=w_{1}(x) & x \in \Omega\end{cases}
$$

with $\Omega$ as above, $\xi_{j} \in \Omega$, and $\lambda_{j} \in H^{2}(0, \tau)$ satisfying $\lambda_{j}(0)=\lambda_{j}^{\prime}(0)=0$ and $\lambda \equiv 0$ after some time $\tau_{1}$. More precisely, we show that the conditions

$$
\frac{\partial w^{(1)}}{\partial \nu}=\frac{\partial w^{(2)}}{\partial \nu} \text { on }(0, \tau) \times \Gamma, \quad \frac{\partial \Delta w^{(1)}}{\partial \nu}=\frac{\partial \Delta w^{(2)}}{\partial \nu} \text { on }\left(0, \tau_{1}\right) \times \Gamma_{1},
$$

with $\Gamma$ as above and $\Gamma_{1}$ a nonempty open subset of $\Gamma$, imply that the completely unknown corresponding sources $\sum_{j=1}^{N^{(1)}} \lambda_{j}^{(1)}(t) \delta_{\xi_{j}^{(1)}}$ and $\sum_{j=1}^{N^{(2)}} \lambda_{j}^{(2)}(t) \delta_{\xi_{j}^{(2)}}$ are equal.

The case of more regular sources, namely $g(t)=\lambda(t) f$, with $f \in H_{0}^{1}(\Omega)$, will be treated in two situations: the usual plate equation (Theorem 4.4) and the plate equation with a potential (Theorem 4.5). In the first one, we establish stability by knowing either $\frac{\partial^{2} w}{\partial t \partial \nu}$ or $\frac{\partial^{2} \Delta w}{\partial t \partial \nu}$. In the last situation, we consider measurements of $\frac{\partial w}{\partial \nu}$ and $\frac{\partial \Delta w}{\partial \nu}$.

In the above applications, the restrictions on the geometry of the domain $\Omega$ are necessary to have the exact observability for the plate equation. We will make use of results due to Lebeau [14] and to Tenenbaum and Tucsnak [21].

In the case where the intensity $\lambda$ in $g(t)=\lambda(t) f$ is known, our method is inspired by the work of Puel and Yamamoto [18] where they consider the wave equation and determine a source which is in $L^{2}(\Omega)$ provided that the domain of $\Gamma$ of observation satisfies the geometric optics condition. Other works treating sources of the form $g(t)=\lambda(t) f$ with $f$ a sum of Dirac masses at points located inside the equations domain are due to Komornik and Yamamoto $[11,12]$ for the wave equation and the heat equation, respectively, and to Nicaise and Zaïr $[16,15]$ for the beam equation and the wave equation in heterogeneous trees, respectively.

As regards the problem of finding more regular sources, we refer to Yamamoto [27, 28] for the wave equation and a source of the form $g(t)=\lambda(t) f$ with $f \in L^{2}(\Omega)$, Wang [24] for the plate equation and a source of the form $g(t)=\lambda(t) f$ with $f \in H_{0}^{1}(\Omega)$. Let us mention that in [24], the condition $\lambda \in C^{3}([0, \tau])$ and of constant sign is needed and the stability result is based on five boundary observations on

$$
\left\{x \in \partial \Omega ;\left(x-x_{0}\right) \cdot \nu(x)>0\right\}, \quad x_{0} \in \mathbb{R}^{2} .
$$


Our contribution in Theorem 4.4 consists in the fact that we obtain a stability result with only two boundary observations and under assumptions which are weaker than those in [24].

Concerning our identifiability result for the problem (1.9) with the number of sources, their location and their intensity all unknown, the approach we use follows the method of El Badia and Ha Duong in [8], which is based on Fourier analysis and appropriate uniqueness results. The identifiability and reconstruction of linear combinations of point sources has also been studied for the heat equation in El Badia and Ha Duong [7] and for the Stokes equations in Alves and Silvestre [2].

The paper is organized as follows. In Section 2, we present some concepts and preliminary results about exact observability and exact controllability, and then collect the available known results concerning the exact observability for the plate equation. Section 3 is devoted to the new results for the stability of sources in the general system (1.1)-(1.3). In Section 4, we apply the results of Section 3 to the plates equation with known intensity and consider the corresponding problem with a potential. Finally, in Section 5, we consider the identifiability of an unknown linear combination of point sources in the plate equation.

\section{Some background on exact observability and exact controllability}

\subsection{Basic concepts and auxiliary results}

In this section we gather, for the convenience of the reader, some known results on functional analysis and, in particular, on exact observability and on exact controllability of infinite dimensional systems. At the end of the section we prove a result, which seems new, concerning an observability inequality involving weakened norms.

The following lemma is a consequence of the closed graph theorem (see, for instance, Douglas [6]).

Lemma 2.1. Suppose that $V_{1}$ and $V_{2}$ are Hilbert spaces, let $V_{1}^{\prime}$ and $V_{2}^{\prime}$ be the corresponding dual spaces and let $F \in \mathcal{L}\left(V_{2}, V_{1}\right)$. Then the following statements are equivalent:

(a) $F$ maps $V_{2}$ onto $V_{1}$;

(b) There exists a constant $c>0$ such that

$$
\left\|F^{*} z\right\|_{V_{2}^{\prime}} \geqslant c\|z\|_{V_{1}^{\prime}} \quad\left(z \in V_{1}^{\prime}\right)
$$

In the next results, the notations $H, H_{1}$ and $H_{2}$ stand for Hilbert spaces which will be identified with their duals. We give below two technical results which are slight variations of those from [23, Section 2.9].

Lemma 2.2. Let $V_{j}, H_{j}, j \in\{1,2\}$ be Hilbert spaces such that $V_{j} \subset H_{j}$ with continuous and dense embeddings. Let $L \in \mathcal{L}\left(H_{1}, H_{2}\right)$ such that $L\left(V_{1}\right) \subset V_{2}$. Then the restriction of $L$ to $V_{1}$ is in $\mathcal{L}\left(V_{1}, V_{2}\right)$.

Proof. We notice that as an operator from $V_{1}$ to $V_{2}, L$ is closed (we have used the continuous embedding of $V_{j}$ into $H_{j}$ for $\left.j \in\{1,2\}\right)$. Therefore, by the closed graph theorem, $L$ is bounded as an operator from $V_{1}$ to $V_{2}$.

Proposition 2.3. Let $V_{j}, H_{j}, j \in\{1,2\}$ be Hilbert spaces such that $V_{j} \subset H_{j}$ with continuous and dense embeddings. Let $L \in \mathcal{L}\left(H_{1}, H_{2}\right)$ be such that $L^{*}\left(V_{2}\right) \subset V_{1}$. Then $L$ can be extended to an

operator $\widetilde{L} \in \mathcal{L}\left(V_{1}^{\prime}, V_{2}^{\prime}\right)$, where $V_{1}^{\prime}$ (respectively $V_{2}^{\prime}$ ) is the dual of $V_{1}$ (respectively of $V_{2}$ ) with respect to the pivot space $H_{1}$ (respectively $\left.H_{2}\right)$. Moreover, if $L^{*}\left(V_{2}\right)=V_{1}$, then there exists $m>0$ such that

$$
\|\widetilde{L} f\|_{V_{2}^{\prime}} \geqslant m\|f\|_{V_{1}^{\prime}} \quad\left(f \in V_{1}^{\prime}\right) .
$$


Proof. To avoid confusion, we use a different notation, namely $L^{d}$, for the restriction of $L^{*}$ to $V_{2}$. We use Lemma 2.2 to conclude that $L^{d} \in \mathcal{L}\left(V_{2}, V_{1}\right)$. Hence, $L^{d *} \in \mathcal{L}\left(V_{1}^{\prime}, V_{2}^{\prime}\right)$. We claim that $L^{d *}$ is an extension of $L$, i.e., that $L^{d *} z=L z$ holds for all $z \in H_{1}$. For this, it will be enough to show that

$$
\left\langle L^{d *} z, \varphi\right\rangle_{V_{2}^{\prime}, V_{2}}=\langle L z, \varphi\rangle_{V_{2}^{\prime}, V_{2}} \quad\left(z \in H_{1}, \varphi \in V_{2}\right) .
$$

Since both the right-hand side and the left-hand side of $(2.11)$ can be written as $\langle L z, \varphi\rangle_{H_{2}}$, the above identity is obviously true. Thus, $\tilde{L}=L^{d *}$ is an extension of $L$.

The uniqueness of $\tilde{L}$ follows from the density of $H_{1}$ in $V_{1}^{\prime}$.

Finally, if $L^{*}\left(V_{2}\right)=V_{1}$ then estimate (2.10) follows by applying Proposition 2.1, with $F=L^{d}$.

We also need the following known result (see, for instance [23, Proposition 2.10.3]).

Proposition 2.4. Let $A: \mathcal{D}(A) \rightarrow X$ be a densely defined operator with resolvent set $\rho(A) \neq \emptyset$, let $\beta \in \rho(A)$, let $X_{1}$ be $\mathcal{D}(A)$ with the graph norm and let $X_{-1}$ be the completion of $X$ with respect to the norm

$$
\|z\|_{-1}=\left\|(\beta I-A)^{-1} z\right\| \quad(z \in X) .
$$

Then $A \in \mathcal{L}\left(X_{1}, X\right)$ and $A$ has a unique extension to an operator in $\mathcal{L}\left(X, X_{-1}\right)$, also denoted by $A$. Moreover,

$$
(\beta I-A)^{-1} \in \mathcal{L}\left(X, X_{1}\right), \quad(\beta I-A)^{-1} \in \mathcal{L}\left(X_{-1}, X\right)
$$

and these two operators are unitary.

Remark 2.5. In the construction of $X_{1}$ we may replace $A$ with $A^{*}$ and $\beta$ with $\bar{\beta}$, obtaining a space denoted $X_{1}^{d}$. Note that $X_{-1}$ is the dual of $X_{1}^{d}$ with respect to the pivot space $X$.

In the sequel, $X, Y$ and $U$ are complex Hilbert spaces which are identified with their duals. $\mathbb{T}=\left(\mathbb{T}_{t}\right)_{t \geqslant 0}$ is a strongly continuous semigroup on $X$, with generator $A: \mathcal{D}(A) \rightarrow X$ and $X_{1}$ is $\mathcal{D}(A)$ with the norm $\|z\|_{1}=\|(\beta I-A) z\|$, where $\beta \in \rho(A)$ is fixed. We denote by $X_{-1}$ the completion of $X$ with respect to the norm $\|z\|_{-1}=\left\|(\beta I-A)^{-1} z\right\|$ and we use the notation $A$ and $\mathbb{T}_{t}$ also for the extension of the original generator to $X$ and for the extension of the original semigroup to $X_{-1}$. Denoting by $A^{*}$ the adjoint of $A, X_{1}^{d}$ is the space $\mathcal{D}\left(A^{*}\right)$ with the norm $\|z\|_{1}^{d}=\left\|\left(\bar{\beta} I-A^{*}\right) z\right\|$ and $X_{-1}^{d}$ is the completion of $X$ with respect to the norm $\|z\|_{-1}^{d}=\left\|\left(\bar{\beta} I-A^{*}\right)^{-1} z\right\|$. Then $X_{-1}$ is the dual of $X_{1}^{d}$ with respect to the pivot space $X$.

Let $C \in \mathcal{L}\left(X_{1}, Y\right)$. For each $\tau>0$, we define the operator $\Psi_{\tau} \in \mathcal{L}\left(X_{1}, L^{2}(0, \tau ; Y)\right)$ by

$$
\left(\Psi_{\tau} z_{0}\right)(t)=C \mathbb{T}_{t} z_{0} \text { for } t \in[0, \tau] \text { and for } z_{0} \in X_{1} \text {. }
$$

Note that, for every $z_{0} \in \mathcal{D}(A)$, we have $\Psi_{\tau} z_{0}=y$, where $z_{0}$ and $y$ are related by (1.4)-(1.5). We next recall a definition which is by now classical in infinite dimensional systems theory (see, for instance, Salamon $[19,20]$, Weiss $[25,26])$.

Definition 2.6. The operator $C \in \mathcal{L}\left(X_{1}, Y\right)$ is called an admissible observation operator for $\mathbb{T}$ if for some (and hence for all) $\tau>0, \Psi_{\tau}$ has a continuous extension to $X$.

Equivalently, the operator $C \in \mathcal{L}\left(X_{1}, Y\right)$ is an admissible observation operator for $\mathbb{T}$ if and only if there exists a positive constant $K_{\tau}$ such that the solution $(z, y)$ of $(1.4)-(1.5)$ satisfies

$$
\int_{0}^{\tau}\|y(t)\|_{Y}^{2} \mathrm{~d} t \leqslant K_{\tau}^{2}\left\|z_{0}\right\|_{X}^{2}, \quad \forall z_{0} \in \mathcal{D}(A)
$$


Definition 2.7. Let $\tau>0$. The pair $(A, C)$ is exactly observable in time $\tau$ if $\Psi_{\tau}$ is bounded from below.

In other words, the pair $(A, C)$ is exactly observable in time $\tau$ if and only if there exists a positive constant $k_{\tau}$ such that the solution $(z, y)$ of $(1.4)-(1.5)$ satisfies

$$
\int_{0}^{\tau}\|y(t)\|_{Y}^{2} \mathrm{~d} t \geqslant k_{\tau}^{2}\left\|z_{0}\right\|_{X}^{2}, \quad \forall z_{0} \in \mathcal{D}(A) .
$$

Let $B \in \mathcal{L}\left(U, X_{-1}\right)$. For each $\tau>0$ we define the operator $\Phi_{\tau} \in \mathcal{L}\left(L^{2}(0, \tau ; U), X_{-1}\right)$ by

$$
\Phi_{\tau} u=\int_{0}^{\tau} \mathbb{T}_{\tau-\sigma} B u(\sigma) \mathrm{d} \sigma
$$

Note that $\Phi_{\tau} u=z(\tau)$ where $z$ is the solution in $X_{-1}$ of the differential equation

$$
\dot{z}(t)=A z(t)+B u(t), \quad z(0)=0 .
$$

Definition 2.8. The operator $B \in \mathcal{L}\left(U ; X_{-1}\right)$ is called an admissible control operator for $\mathbb{T}$ if for some (and hence for all) $\tau>0, \operatorname{Ran} \Phi_{\tau} \subset X$.

From Lemma 2.2 , if $B \in \mathcal{L}\left(U, X_{-1}\right)$ is admissible then for every $\tau \geqslant 0$ we have

$$
\Phi_{\tau} \in \mathcal{L}\left(L^{2}(0, \tau ; U), X\right) .
$$

Definition 2.9. Let $\tau>0$. The pair $(A, B)$ is exactly controllable in time $\tau$ if $\operatorname{Ran} \Phi_{\tau}=X$.

If $B \in \mathcal{L}\left(U, X_{-1}\right)$ then, using the duality between $X_{1}^{d}$ (which is $\mathcal{D}\left(A^{*}\right)$ with the graph norm) and $X_{-1}$ and identifying $U$ with its dual, we have $B^{*} \in \mathcal{L}\left(X_{1}^{d}, U\right)$. The adjoint of $\Phi_{\tau}$ from (2.14), which is in $\mathcal{L}\left(X_{1}^{d}, L^{2}(0, \infty ; U)\right)$, can be expressed using $B^{*}$ and the exact controllability of the pair $(A, B)$ is equivalent to the exact observability of the pair $\left(A^{*}, B^{*}\right)$. This type of results goes back to Dolecki and Russell [5] and we state them below in a form borrowed from [23, Proposition 4.4.1, Theorem 10.2.1]

Proposition 2.10. If $B \in \mathcal{L}\left(U, X_{-1}\right)$, then for every $\tau>0$ and every $z_{0} \in X_{1}^{d}$,

$$
\left(\Phi_{\tau}^{*} z_{0}\right)(t)= \begin{cases}B^{*} \mathbb{T}_{\tau-t}^{*} z_{0} & \text { for } t \in[0, \tau], \\ 0 & \text { for } t>\tau .\end{cases}
$$

If $B$ is an admissible control operator for $\mathbb{T}$, so that $\Phi_{\tau}$ can also be regarded as an operator in $\mathcal{L}\left(L^{2}(0, \infty ; U), X\right)$, then its adjoint in $\mathcal{L}\left(X, L^{2}(0, \infty ; U)\right)$ is given, for $z_{0} \in \mathcal{D}\left(A^{*}\right)$, by the same formula (2.15).

Proposition 2.11. Suppose that $B \in \mathcal{L}\left(U, X_{-1}\right)$. Then $B$ is an admissible control operator for $\mathbb{T}$ if and only if $B^{*}$ is an admissible observation operator for $\mathbb{T}^{*}$.

The pair $(A, B)$ is exactly controllable in time $\tau$ if and only if $\left(A^{*}, B^{*}\right)$ is exactly observable in time $\tau$.

If a pair $(A, B)$ is exactly controllable in a time $\tau_{0}$, a natural question is the characterization of the states which can be reached by more regular inputs. Before stating a result in this direction, we introduce additional notations. For a Hilbert space $V$, and for $\tau>0$ we set

$$
\begin{aligned}
& H_{L}^{1}(0, \tau ; V)=\left\{u \in H^{1}(0, \tau ; V) \mid u(0)=0\right\}, \\
& H_{R}^{1}(0, \tau ; V)=\left\{u \in H^{1}(0, \tau ; V) \mid u(\tau)=0\right\} .
\end{aligned}
$$

The following result has been proved in Tucsnak and Weiss [22] in the case of a finite dimensional input space $U$ and in the general form below in [23, Theorem 10.3.6]. 
Proposition 2.12. Suppose that $B \in \mathcal{L}\left(U, X_{-1}\right)$ is an admissible control operator for $\mathbb{T}$. Then for all $\tau>0$

$$
\Phi_{\tau} \in \mathcal{L}\left(H_{L}^{1}(0, \tau ; U), Z\right)
$$

where

$$
Z=X_{1}+(\beta I-A)^{-1} B U=(\beta I-A)^{-1}(X+B U)
$$

for some $\beta \in \rho(A)$.

Suppose moreover that the pair $(A, B)$ is exactly controllable in time $\tau_{0}$. Then for all $\tau>\tau_{0}, \Phi_{\tau}$ is onto from $H_{L}^{1}(0, \tau ; U)$ to $Z$ and there exists $M_{\tau}>0$ such that, for every $z_{0} \in Z$, the minimal norm control $u$ with $\Phi_{\tau} u=z_{0}$ satisfies

$$
\|u\|_{H_{L}^{1}(0, \tau ; U)} \leqslant M_{\tau}\left\|z_{0}\right\|_{Z}
$$

Remark 2.13. Note that the space $Z$ defined by (2.16) does not depend on the choice of $\beta$. The norm of $Z$ is defined by

$$
\|z\|_{Z}^{2}=\inf \left\{\|x\|_{X}^{2}+\|u\|_{U}^{2} ; x \in X, u \in U, z=(\beta I-A)^{-1}(x+B u)\right\} .
$$

If $E$ is a dense subspace of $X$ we denote by $E^{\prime}$ the dual of $E$ with respect to the pivot space $X$. Moreover, we denote by $\left[H_{L}^{1}(0, \tau ; U)\right]^{\prime}$ (respectively by $\left[H_{R}^{1}(0, \tau ; U)\right]^{\prime}$ ) the dual of $H_{L}^{1}(0, \tau ; U)$ (respectively $H_{R}^{1}(0, \tau ; U)$ ) with respect to the pivot space $L^{2}(0, \tau ; U)$.

Let $(A, C)$ be exactly observable in time $\tau_{0}$. Another natural question, which is dual to that in Proposition 2.12, is to find a lower bound of $\left\|\Psi_{\tau} z_{0}\right\|_{w}$, where $\|\cdot\|_{w}$ is a norm which is weaker than the norm in $L^{2}(0, \tau ; Y)$. A partial answer is given by the result below.

Proposition 2.14. Let $A: \mathcal{D}(A) \rightarrow X$ be a densely defined operator and let $C \in \mathcal{L}\left(X_{1}, Y\right)$ be an admissible observation operator for $\mathbb{T}$. For $\tau>0$ let $\Psi_{\tau}$ be the output map corresponding to the pair $(A, C)$, as defined in (2.13). Then, for each $\tau>0, \Psi_{\tau}$ has a unique continuous extension $\Psi_{\tau} \in \mathcal{L}\left(\left(Z^{d}\right)^{\prime},\left[H_{R}^{1}(0, \tau ; Y)\right]^{\prime}\right)$, where

$$
Z^{d}=\left(\beta I-A^{*}\right)^{-1}\left(X+C^{*} Y\right) .
$$

Moreover, assume that $(A, C)$ is exactly observable in some time $\tau_{0}>0$. Then, for each $\tau>\tau_{0}$, there exists a constant $m_{\tau}>0$ such that, for every $f \in\left(Z^{d}\right)^{\prime}$, we have

$$
\left\|\Psi_{\tau} f\right\|_{\left[H_{R}^{1}(0, \tau ; Y)\right]^{\prime}} \geqslant m_{\tau}\|f\|_{\left(Z^{d}\right)^{\prime}} .
$$

Proof. Since $C$ is an admissible observation operator for $\mathbb{T}$, Proposition 2.11 yields that $C^{*}$ is an admissible control operator for $\mathbb{T}^{*}$. By applying Proposition 2.12 , it follows that $\Phi_{\tau}^{d}$ maps $H_{L}^{1}(0, \tau ; Y)$ to $Z^{d}$. As a consequence $\Phi_{\tau}^{d} \boldsymbol{Я}_{\tau}$ maps $H_{R}^{1}(0, \tau ; Y)$ to $Z^{d}$, with $\boldsymbol{Я}_{\tau}$ defined by

$$
\left(\boldsymbol{Я}_{\tau} f\right)(s)=f(\tau-s) \quad(s \in[0, \tau]) .
$$

On the other hand, by using Proposition 2.10 we have

$$
\Phi_{\tau}^{d} \boldsymbol{Я}_{\tau}=\Psi_{\tau}^{*}
$$

so that the conclusion follows from Proposition 2.3.

If $(A, C)$ is exactly observable in time $\tau_{0}$, we can apply Proposition 2.11 to obtain that $\left(A^{*}, C^{*}\right)$ is exactly controllable in time $\tau_{0}$. Let $\tau>\tau_{0}$. By applying again Proposition 2.12, it follows that $\Phi_{\tau}^{d}$ maps $H_{L}^{1}(0, \tau ; Y)$ onto $Z^{d}$ and the conclusion follows again from Proposition 2.3.

Remark 2.15. In (2.18), $A^{*}$ is extended to $X$ as a skew-adjoint operator from $X$ to $X_{-1}^{d}$ (the completed space of $X$ for the norm $z \mapsto\left\|\left(\beta I-A^{*}\right)^{-1} z\right\|$ (see Proposition 2.4). 


\subsection{Exact observability for the plate equation}

In this subsection, we collect some known results for the Euler-Bernoulli plate equation and obtain a new result concerning the observability for a problem with a potential term.

Let $\Omega$ be a smooth domain of $\mathbb{R}^{2}$ and $\Gamma$ a nonempty open subset of $\partial \Omega$. We first assume that $\Gamma$ satisfies the following geometric optics condition: for all $x \in \Omega$, any ray coming from $x$ at initial time, propagating at velocity one and following the geometric optics laws, meets $\bar{\Gamma}$ in finite time.

This condition is sufficient and almost necessary to have the exact observability of the wave equation. Lebeau proved in [14] that this condition is also sufficient for the plate equation

$$
\begin{cases}\frac{\partial^{2} w}{\partial t^{2}}+\Delta^{2} w=0, & \text { in }(0, \tau) \times \Omega \\ w=\Delta w=0 & \text { on }(0, \tau) \times \partial \Omega \\ w(0, x)=w_{0}(x), \frac{\partial w}{\partial t}(0, x)=w_{1}(x) & x \in \Omega .\end{cases}
$$

More precisely, the following result has been proved in [14].

Theorem 2.16. Let $\tau>0$, let $\Omega$ be a smooth bounded domain of $\mathbb{R}^{2}$ and assume that $\Gamma$ satisfies the geometric optics condition. Then, for any initial data

$$
\begin{gathered}
w_{0} \in H^{5}(\Omega), w_{0}=\Delta w_{0}=\Delta^{2} w_{0}=0 \text { on } \partial \Omega, \text { and } \\
w_{1} \in H^{3}(\Omega), w_{1}=\Delta w_{1}=0 \text { on } \partial \Omega,
\end{gathered}
$$

the system (2.20) has a unique solution $w \in C^{0}\left([0, \tau] ; H^{5}(\Omega)\right) \cap C^{1}\left([0, \tau] ; H^{3}(\Omega)\right)$ that satisfies

$$
\int_{0}^{\tau}\left\|\frac{\partial^{2} w}{\partial t \partial \nu}\right\|_{L^{2}(\Gamma)}^{2} \mathrm{~d} t \geqslant k_{\tau}^{2}\left(\left\|w_{0}\right\|_{H^{3}(\Omega)}^{2}+\left\|w_{1}\right\|_{H^{1}(\Omega)}^{2}\right) .
$$

More recently, in [21], it has been proved that the geometric optics condition is not necessary in the case of a rectangle. More precisely a result in [21] states that:

Theorem 2.17. Let $\tau>0$ and let $\Omega=(0, a) \times(0, b)(a, b>0)$. Assume that $\Gamma$ is an open subset of $\partial \Omega$ containing both a horizontal and a vertical segment of nonzero length. Then, for any

$$
\begin{gathered}
w_{0} \in H^{5}(\Omega), w_{0}=\Delta w_{0}=\Delta^{2} w_{0}=0 \text { on } \partial \Omega, \quad \text { and } \\
w_{1} \in H^{3}(\Omega), w_{1}=\Delta w_{1}=0 \text { on } \partial \Omega,
\end{gathered}
$$

the system (2.20) has a unique solution $w \in C^{0}\left([0, \tau] ; H^{5}(\Omega)\right) \cap C^{1}\left([0, \tau] ; H^{3}(\Omega)\right)$ that satisfies

$$
\int_{0}^{\tau}\left\|\frac{\partial^{2} w}{\partial t \partial \nu}\right\|_{L^{2}(\Gamma)}^{2} \mathrm{~d} t \geqslant k_{\tau}^{2}\left(\left\|w_{0}\right\|_{H^{3}(\Omega)}^{2}+\left\|w_{1}\right\|_{H^{1}(\Omega)}^{2}\right) .
$$

Moreover, the above inequality is false if $\Gamma$ does not contain a vertical or an horizontal subset.

Using classical arguments, we deduce from the two above theorems the following observability result:

Corollary 2.18. Assume that $\Omega$ is a bounded domain of $\mathbb{R}^{2}$ and $\Gamma$ is an open subset of $\partial \Omega$ such that one of the following assertions holds: 
1. $\partial \Omega$ is smooth and $\Gamma$ satisfies the geometric optics conditions;

2. $\Omega$ is a rectangle $(0, a) \times(0, b)$ and $\Gamma$ contains both a horizontal and a vertical segment of nonzero length.

Suppose that

$$
\begin{gathered}
w_{0} \in H^{5}(\Omega), w_{0}=\Delta w_{0}=\Delta^{2} w_{0}=0 \text { on } \partial \Omega, \text { and } \\
w_{1} \in H^{3}(\Omega), w_{1}=\Delta w_{1}=0 \text { on } \partial \Omega .
\end{gathered}
$$

Then, the solution $w \in C^{0}\left([0, \tau] ; H^{5}(\Omega)\right) \cap C^{1}\left([0, \tau] ; H^{3}(\Omega)\right)$ of the system (2.20) satisfies

$$
\int_{0}^{\tau}\left\|\frac{\partial \Delta w}{\partial \nu}\right\|_{L^{2}(\Gamma)}^{2} \mathrm{~d} t \geqslant k_{\tau}\left(\left\|w_{0}\right\|_{H^{3}(\Omega)}^{2}+\left\|w_{1}\right\|_{H^{1}(\Omega)}^{2}\right) .
$$

Now we consider the exact observability of the problem with potential

$$
\begin{cases}\frac{\partial^{2} w}{\partial t^{2}}+\Delta^{2} w+d w=0, & \text { in }(0, \tau) \times \Omega \\ w=\Delta w=0 & \text { on }(0, \tau) \times \partial \Omega \\ w(0, x)=w_{0}(x), \frac{\partial w}{\partial t}(0, x)=w_{1}(x) & x \in \Omega\end{cases}
$$

with $d \in H^{1+\alpha}(\Omega)$, with $\alpha>0$. We can deduce from Theorems 2.16 and 2.17 the following result.

Theorem 2.19. Let $\Omega$ and $\Gamma$ satisfy the assumptions of Corollary 2.18. Assume that $\Gamma_{1}$ is an open nonempty subset of $\Gamma$. and assume that $d \in H^{1+\alpha}(\Omega)$, with $\alpha>0$. Then, for any

$$
\begin{gathered}
w_{0} \in H^{5}(\Omega), w_{0}=\Delta w_{0}=\Delta^{2} w_{0}=0 \text { on } \partial \Omega, \text { and } \\
w_{1} \in H^{3}(\Omega), w_{1}=\Delta w_{1}=0 \text { on } \partial \Omega,
\end{gathered}
$$

the system $(2.21)$ has a unique solution $w \in C^{0}\left([0, \tau] ; H^{5}(\Omega)\right) \cap C^{1}\left([0, \tau] ; H^{3}(\Omega)\right)$ and this solution satisfies

$$
\int_{0}^{\tau}\left(\left\|\frac{\partial \Delta w}{\partial \nu}\right\|_{L^{2}(\Gamma)}^{2}+\left\|\frac{\partial w}{\partial \nu}\right\|_{L^{2}\left(\Gamma_{1}\right)}^{2}\right) \mathrm{d} t \geqslant k_{\tau}\left(\left\|w_{0}\right\|_{H^{3}(\Omega)}^{2}+\left\|w_{1}\right\|_{H^{1}(\Omega)}^{2}\right) .
$$

To prove Theorem 2.19, we use a strategy borrowed from [23, Section 6.3]. Let us first set

$$
\begin{gathered}
H=H_{0}^{1}(\Omega), \quad \mathcal{D}\left(A_{0}\right)=\left\{\varphi \in H^{5}(\Omega) \mid \varphi=\Delta \varphi=\Delta^{2} \varphi=0 \text { on } \partial \Omega\right\}, \\
Y=L^{2}(\Gamma) \times L^{2}\left(\Gamma_{1}\right), \quad A_{0} \varphi=\Delta^{2} \varphi \quad \forall \varphi \in \mathcal{D}\left(A_{0}\right) .
\end{gathered}
$$

Then we have

$$
\mathcal{D}\left(A_{0}^{1 / 2}\right)=\left\{\varphi \in H^{3}(\Omega) \mid \varphi=\Delta \varphi=0 \text { on } \partial \Omega\right\}
$$

and if we set

$$
\begin{gathered}
X=\mathcal{D}\left(A_{0}^{1 / 2}\right) \times H, \quad \mathcal{D}(A)=\mathcal{D}\left(A_{0}\right) \times \mathcal{D}\left(A_{0}^{1 / 2}\right), \\
A=\left[\begin{array}{cc}
0 & I \\
-A_{0} & 0
\end{array}\right],
\end{gathered}
$$


the operator $A$ is skew-adjoint and thus, by Stone's theorem, is the generator of a strongly continuous group of isometries $\mathbb{T}$ in $X$. The operator $C \in \mathcal{L}(\mathcal{D}(A), Y)$ is defined by

$$
C\left[\begin{array}{l}
\varphi \\
\psi
\end{array}\right]=\left[\begin{array}{c}
\frac{\partial \varphi}{\partial \nu} \\
\frac{\partial\left(A_{0}^{1 / 2} \varphi\right)}{\partial \nu}
\end{array}\right] \quad\left(\left[\begin{array}{l}
\varphi \\
\psi
\end{array}\right] \in \mathcal{D}(A)\right)
$$

and it can be checked that $C$ is an admissible operator for $\mathbb{T}$.

We also define the following operators:

$$
\begin{gathered}
P_{0} \in \mathcal{L}\left(\mathcal{D}\left(A_{0}^{1 / 2}\right), H\right), \quad P_{0} f=-d f \quad(f \in H), \\
P=\left[\begin{array}{cc}
0 & 0 \\
P_{0} & 0
\end{array}\right] \in \mathcal{L}(X), \\
A_{P}=A+P \text { with } \mathcal{D}\left(A_{P}\right)=\mathcal{D}(A) .
\end{gathered}
$$

Since $A_{P}$ is a bounded perturbation of $A$, according to [17, Theorem 1.1, p.76], $A_{P}$ is the generator of a strongly continuous semigroup $\mathbb{T}_{P}$ on $X$. We remark that $P_{0} \in \mathcal{L}(H)$. Moreover, we can check that $A_{0}-P_{0}$ is a self-adjoint operator with compact resolvents. In particular, it is diagonalisable with an orthonormal basis $\left(\varphi_{k}\right)_{k \in \mathbb{N}^{*}}$ of eigenvectors and the corresponding family of real eigenvalues $\left(\lambda_{k}\right)_{k \in \mathbb{N}^{*}}$ satisfies $\lambda_{k}>-\left\|P_{0}\right\|_{\mathcal{L}(H)}$ for all $k \in \mathbb{N}^{*}$, and

$$
\lim _{k \rightarrow \infty} \lambda_{k}=+\infty
$$

We can assume that the sequence $\left(\lambda_{k}\right)_{k \in \mathbb{N}^{*}}$ is non decreasing. We extend the sequence $\left(\varphi_{k}\right)_{k \in \mathbb{N}^{*}}$ to a sequence indexed by $\mathbb{Z}^{*}$ by setting $\varphi_{k}=-\varphi_{-k}$ for every $k \in \mathbb{Z}_{-}^{*}$. We introduce the real sequence $\left(\mu_{k}\right)_{k \in \mathbb{Z}^{*}}$ by

$$
\mu_{k}=\sqrt{\left|\lambda_{k}\right|} \text { if } k>0 \text { and } \mu_{k}=-\mu_{-k} \text { if } k<0 .
$$

We denote

$$
\begin{gathered}
W_{0}=\operatorname{span}\left\{\left[\begin{array}{c}
\frac{1}{i \operatorname{sgn}(k)} \varphi_{k} \\
\varphi_{k}
\end{array}\right] ; k \in \mathbb{Z}^{*}, \mu_{k}=0\right\}, \\
W_{N}=\operatorname{span}\left\{\left[\begin{array}{c}
\frac{1}{i \mu_{k}} \varphi_{k} \\
\varphi_{k}
\end{array}\right] ; k \in \mathbb{Z}^{*},|k|<N, \mu_{k} \neq 0\right\},
\end{gathered}
$$

with $N \in \mathbb{N}^{*}$ such that $\lambda_{N}>0$, and

$$
Y_{N}=W_{0}+W_{N}
$$

We also set

$$
V_{N}=\operatorname{clos} \operatorname{span}\left\{\left[\begin{array}{c}
\frac{1}{i \mu_{k}} \varphi_{k} \\
\varphi_{k}
\end{array}\right] ; k \in \mathbb{Z}^{*},|k| \geqslant N\right\}
$$

It is not difficult to check that $X=Y_{N} \oplus V_{N}$ and that $Y_{N}$ and $V_{N}$ are invariant under $\mathbb{T}_{P}$. Let us consider the restriction $P_{V_{N}} \in \mathcal{L}\left(V_{N}, X\right)$ of $P$ to $V_{N}$. We have

Lemma 2.20. Let $\varepsilon>0$. Then there exists a positive constant $K$ depending on $\Omega$ and on $\varepsilon$ such that

$$
\left\|P_{V_{N}}\right\|_{\mathcal{L}\left(V_{N}, X\right)} \leqslant K \frac{\|d\|_{H^{1}(\Omega)}}{\lambda_{N}^{1 / 4-\varepsilon}} .
$$


Proof. Assume that $z=\left[\begin{array}{l}f \\ g\end{array}\right] \in X$. Then

$$
\left\|P_{V_{N}} z\right\|_{X}=\|d f\|_{H_{0}^{1}(\Omega)} .
$$

Using Hölder's inequality, we deduce from the above inequality

$$
\left\|P_{V_{N}} z\right\|_{X} \leqslant\|d\|_{H^{1}(\Omega)}\|f\|_{W^{1, \infty}(\Omega)} .
$$

From Sobolev embedding theorem, we deduce that for $\varepsilon>0$, there exists a positive constant $K$ such that

$$
\left\|P_{V_{N}} z\right\|_{X} \leqslant K\|d\|_{H^{1}(\Omega)}\|f\|_{H^{2+4 \varepsilon}(\Omega)} .
$$

On the other hand, there exists a positive constant $K$ such that

$$
\|f\|_{H^{2+4 \varepsilon}(\Omega)} \leqslant K\|f\|_{D\left(A_{0}^{1 / 4+\varepsilon}\right)}=K\|f\|_{D\left(A_{P}^{1 / 4+\varepsilon}\right)} \cdot
$$

In particular, if $z \in V_{N}$, then

$$
\|f\|_{H^{2+4 \varepsilon}(\Omega)} \leqslant K \frac{1}{\left|\lambda_{N}\right|^{1 / 4-\varepsilon}}\|f\|_{D\left(A_{P}^{1 / 2}\right)}=K \frac{1}{\left|\lambda_{N}\right|^{1 / 4-\varepsilon}}\|f\|_{D\left(A_{0}^{1 / 2}\right)} .
$$

The above equation and (2.22) yield the desired result.

We are now in position to prove Theorem 2.19:

Proof of Theorem 2.19. We know from Corollary $2.18\left(\left.A\right|_{V_{N}}, C_{N}\right)$ is exactly observable in any time $\tau>0$. Denote $A_{N}=\left.A_{P}\right|_{V_{N}}$ and $C_{N}=\left.C\right|_{V_{N}}$. By using the fact that, according to Lemma 2.20 we have

$$
\lim _{N \rightarrow \infty}\left\|P_{V_{N}}\right\|_{\mathcal{L}\left(V_{N}, X\right)}=0
$$

and Proposition 5.3.3 from [23] it follows that the pair $\left(A_{N}, C_{N}\right)$ is also exactly observable for all $\tau>0$, provided that $N$ is large enough.

On the other hand, if $\phi=\left[\begin{array}{l}\varphi \\ \psi\end{array}\right] \in D\left(A_{P}\right)$ is an eigenvector of $A_{P}$ associated to the eigenvalue $i \mu$ such that $C \phi=0$ then an easy calculation shows that $\varphi \in D\left(A_{0}\right)$ is an eigenvector of $A_{0}-P_{0}$ associated to the eigenvalue $\mu^{2}$ :

$$
\begin{gathered}
\Delta^{2} \varphi+d \varphi=\mu^{2} \varphi \quad \text { in } \Omega, \\
\varphi=0 \quad \text { and } \quad \Delta \varphi=0 \quad \text { on } \partial \Omega .
\end{gathered}
$$

Moreover the condition $C \phi=0$ implies that

$$
\frac{\partial \varphi}{\partial \nu}=0 \quad \text { and } \quad \frac{\partial \Delta \varphi}{\partial \nu}=0 \quad \text { on } \Gamma_{1}
$$

From a unique continuation result for the bilaplacian, we deduce that $\varphi=0$ and therefore that $\phi=0$. By Hautus lemma (see [9]), we conclude that $\left(\left.A_{P}\right|_{Y_{N}},\left.C\right|_{Y_{N}}\right)$ is exactly observable for all $\tau>0$. Finally, since $A_{N}$ and $\left.A_{P}\right|_{Y_{N}}$ have no common eigenvalues, we can apply Theorem 3.3 of [22] to deduce that $\left(A_{P}, C\right)$ is exactly observable in any time $\tau>0$. 


\section{Stability for an inverse source problem with known intensity}

Throughout this section we continue to use notation introduced in the previous ones. More precisely, $X, Y$ are Hilbert spaces, $A: \mathcal{D}(A) \rightarrow X$ is the generator of a strongly continuous semigroup $\mathbb{T}$ on $X$ and $C \in \mathcal{L}(\mathcal{D}(A), Y)$ is an admissible observation operator for $\mathbb{T}$.

We consider the differential equation

$$
\dot{z}(t)=A z(t)+\lambda(t) f, \quad z(0)=z_{0}
$$

where $z_{0} \in X$ and $f \in Z^{\prime}$, with $Z=\left(\beta I-A^{*}\right)^{-1}\left(X+C^{*} Y\right)$. Assume $\tau>0$ and that we are measuring

$$
y(t)=C z(t) \quad(t \in[0, \tau]) .
$$

Our aim is to study the mapping $f \mapsto y$, assuming that $\lambda$ and $z_{0}$ are given. It is convenient to recall that, in the case where $f \in X$ and $z_{0} \in \mathcal{D}(A)$, the solution of $(3.1)$ satisfies $z \in C^{0}([0, \tau] ; \mathcal{D}(A)) \cap$ $C^{1}([0, \tau] ; X)$ and, by Duhamel formula, $y$ satisfies

$$
y(t)=\int_{0}^{t} \lambda(t-s) C \mathbb{T}_{s} f \mathrm{~d} s+C \mathbb{T}_{t} z_{0}=\int_{0}^{t} \lambda(t-s) \Psi_{\tau} f(s) \mathrm{d} s+\Psi_{\tau} z_{0}(t) .
$$

Proposition 3.1. Let $\tau>0$, let $Y$ be a Hilbert space and $\lambda \in H^{1}(0, \tau)$ with $\lambda(0) \neq 0$. Let $S$ : $L^{2}(0, \tau ; Y) \rightarrow H_{L}^{1}(0, \tau ; Y)$ be defined by

$$
(S g)(t)=\int_{0}^{t} \lambda(t-s) g(s) \mathrm{d} s .
$$

Then $S$ is an isomorphism from $L^{2}(0, \tau ; Y)$ onto $H_{L}^{1}(0, \tau ; Y)$. Moreover, the operator $S$ admits a unique extension to an isomorphism $\widetilde{S}$ from $\left[H_{R}^{1}(0, \tau ; Y)\right]^{\prime}$ onto $L^{2}(0, \tau ; Y)$.

Proof. The fact that $S$ is an isomorphism is well-known from the the theory of Volterra integral operators (see, for instance, Kress [13, pp.33-34]). Denote $\mathcal{X}=L^{2}(0, \tau ; Y), \mathcal{X}_{1}=H_{L}^{1}(0, \tau ; Y)$ and let $\mathcal{A} \in \mathcal{L}\left(\mathcal{X}_{1}, \mathcal{X}\right)$ be the inverse of $S$. Then $\mathcal{A}$ can be seen as an unbounded densely defined operator in $\mathcal{X}$ so that $\mathcal{A}^{*}=\left(S^{*}\right)^{-1}$. It is easy to check that $S^{*}$ maps $L^{2}(0, \tau ; Y)$ onto $H_{R}^{1}(0, \tau ; Y)$ so that $\mathcal{D}\left(\mathcal{A}^{*}\right)=H_{R}^{1}(0, \tau ; Y)$. By applying Proposition 2.4 and Remark 2.5 to $\mathcal{A}$, we obtain that $\mathcal{A}$ has a unique extension to an isomorphism $\widetilde{\mathcal{A}} \in \mathcal{L}\left(L^{2}(0, \tau ; Y),\left[H_{R}^{1}(0, \tau ; Y)\right]^{\prime}\right)$. Consequently, $\widetilde{S}:=\widetilde{\mathcal{A}}^{-1}$ is an isomorphism from $\left[H_{R}^{1}(0, \tau ; Y)\right]^{\prime}$ onto $L^{2}(0, \tau ; Y)$ and it is an extension of $S$.

Now we can show that for less regular data, the mapping $f \mapsto y$ associated with system (3.1)-(3.2) is still well defined.

Proposition 3.2. Assume that $\lambda \in H^{1}(0, \tau), \lambda(0) \neq 0$. Assume that $f \in Z^{\prime}$ and that $z_{0} \in X$. Then equation (3.1) admits a unique solution $z \in C^{0}([0, \tau] ; X)$ such that $y \in L^{2}(0, \tau ; Y)$.

Proof. The first conclusion follows from [23, Theorem 4.1.6], by using the fact that the right-hand side of (3.1) belongs to $H^{1}\left(0, \tau ; X_{-1}\right)$

On the other hand, $y=\left(\widetilde{S} \circ \Psi_{\tau}\right) f+\Psi_{\tau} z_{0} \in L^{2}(0, \tau ; Y)$ where $\widetilde{S}:\left[H_{R}^{1}(0, \tau ; Y)\right]^{\prime} \rightarrow L^{2}(0, \tau ; Y)$ is the extension of $S$ defined in Proposition 3.1 and $\Psi_{\tau}: Z^{\prime} \rightarrow\left[H_{R}^{1}(0, \tau ; Y)\right]^{\prime}$ is defined in Proposition 2.14 . 
In order to study the stability for the inverse source problem, we have to consider two sources $f^{(1)}$ and $f^{(2)}$ and the corresponding solutions $z^{(1)}$ and $z^{(2)}$ and observations $y^{(1)}$ and $y^{(2)}$. Due to the linearity of the problem, it is enough to consider the system

$$
\begin{gathered}
\dot{z}(t)=A z(t)+\lambda(t) f, \quad z(0)=0, \\
y(t)=C z(t) \quad(t \in[0, \tau]) .
\end{gathered}
$$

Assume that $C \in \mathcal{L}\left(X_{1}, Y\right)$ is an admissible observation operator for $\mathbb{T}$ and that $\lambda \in H^{1}(0, \tau)$ with $\lambda(0) \neq 0$. For each $\tau>0$, we introduce the operator $\mathbb{E}_{\tau} \in \mathcal{L}\left(X, H_{L}^{1}(0, \tau ; Y)\right)$ defined by

$$
\left(\mathbb{E}_{\tau} f\right)(t)=\left[\left(S \circ \Psi_{\tau}\right) f\right](t)=\int_{0}^{t} \lambda(t-s) \Psi_{\tau} f(s) \mathrm{d} s \quad(t \in[0, \tau]) .
$$

By using Proposition 3.1 we extend $\mathbb{E}_{\tau}$ to an operator $\mathbb{F}_{\tau} \in \mathcal{L}\left(Z^{\prime}, L^{2}(0, \tau ; Y)\right)$ defined by

$$
\left(\mathbb{F}_{\tau} f\right)(t)=\left[\left(\widetilde{S} \circ \Psi_{\tau}\right) f\right](t) \quad(t \in[0, \tau]),
$$

where $\widetilde{S}$ is the operator constructed in Proposition 3.1. The main result of this section is

Theorem 3.3. Let $X, Y$ be Hilbert spaces and assume that the pair $(A, C)$ is exactly observable in some time $\tau_{0}>0$ and that $\lambda \in H^{1}(0, \tau)$ with $\lambda(0) \neq 0$. Then, the following properties hold:

1. for every $\tau \geqslant \tau_{0}, \mathbb{E}_{\tau}$ is one-to-one from $X$ to $H_{L}^{1}(0, \tau ; Y)$ and there exists a positive constant $\kappa_{\tau}$ such that

$$
\|f\|_{X} \leqslant \kappa_{\tau}\left\|\mathbb{E}_{\tau} f\right\|_{H_{L}^{1}(0, \tau ; Y)}, \quad \forall f \in X
$$

2. for every $\tau>\tau_{0}, \mathbb{F}_{\tau}$ is one-to-one from $Z^{\prime}$ to $L^{2}(0, \tau ; Y)$ and there exists a positive constant $\tilde{\kappa}_{\tau}$ such that

$$
\|f\|_{Z^{\prime}} \leqslant \tilde{\kappa}_{\tau}\left\|\mathbb{F}_{\tau} f\right\|_{L^{2}(0, \tau ; Y)}, \quad \forall f \in Z^{\prime} .
$$

Proof. In the first case, since $\lambda(0) \neq 0, S: L^{2}(0, \tau ; Y) \rightarrow H_{L}^{1}(0, \tau ; Y)$ is an isomorphism and we have

$$
\|\mathbb{E} f\|_{H_{L}^{1}(0, \tau ; Y)}=\left\|\left(S \circ \Psi_{\tau}\right) f\right\|_{H_{L}^{1}(0, \tau ; Y)} \geqslant M_{S}\left\|\Psi_{\tau} f\right\|_{L^{2}(0, \tau ; Y)} .
$$

From the exact observability of $(A, C)$ in time $\tau$, we deduce

$$
\left\|\Psi_{\tau} f\right\|_{L^{2}(0, \tau ; Y)} \geqslant k_{\tau}\|f\|_{X}
$$

Combining the two above inequalities yields

$$
\|\mathbb{E} f\|_{H_{L}^{1}(0, \tau ; Y)} \geqslant \kappa_{\tau}\|f\|_{X} \quad(f \in X) .
$$

For the second case the proof is similar. By using Propositions 3.1 and 2.14, we have

$$
\|\mathbb{F} f\|_{L^{2}(0, \tau ; Y)}=\left\|\left(\widetilde{S} \circ \Psi_{\tau}\right) f\right\|_{L^{2}(0, \tau ; Y)} \geqslant M_{\widetilde{S}}\left\|\Psi_{\tau} f\right\|_{\left[H_{R}^{1}(0, \tau ; Y)\right]^{\prime}}
$$

and

$$
\left\|\Psi_{\tau} f\right\|_{\left[H_{R}^{1}(0, \tau ; Y)\right]^{\prime}} \geqslant m_{\tau}\|f\|_{Z^{\prime}}
$$

Thus,

$$
\|\mathbb{F} f\|_{L^{2}(0, \tau ; Y)} \geqslant M_{\widetilde{S}} m_{\tau}\|f\|_{Z^{\prime}} \quad\left(f \in Z^{\prime}\right) .
$$




\section{Inverse source problems for the plate equation with known inten- sity}

In this section we apply the general results obtained in the previous sections to the inverse source problem for the plate equation, assuming that the intensity $\lambda$ is known and that the domain $\Omega$ satisfies the geometric conditions already mentioned.

\subsection{Recovery of point sources}

We first introduce some notation. Consider the following Hilbert spaces

$$
X=H_{0}^{1}(\Omega) \times H^{-1}(\Omega), \quad W=\left\{\varphi \in H^{3}(\Omega) \mid \varphi=\Delta \varphi=0 \text { on } \partial \Omega\right\}
$$

and the skew-adjoint operator defined by

$$
\begin{aligned}
& \mathcal{D}(A)=W \times H_{0}^{1}(\Omega) \\
& A\left[\begin{array}{l}
\varphi \\
\psi
\end{array}\right]=\left[\begin{array}{c}
\psi \\
-\Delta^{2} \varphi
\end{array}\right] \quad\left(\left[\begin{array}{l}
\varphi \\
\psi
\end{array}\right] \in \mathcal{D}(A)\right) .
\end{aligned}
$$

It is easy to check that the dual of $X_{1}$ (i.e., of $\mathcal{D}(A)$ endowed with the graph topology) with respect to the pivot space $X$ is

$$
X_{-1}=H^{-1}(\Omega) \times W^{\prime},
$$

where $W^{\prime}$ is the dual of $W$ with respect to the pivot space $L^{2}(\Omega)$.

Let $\Gamma$ be an open subset of $\partial \Omega$, denote $Y=L^{2}(\Gamma)$ and let $C \in \mathcal{L}\left(X_{1}, Y\right)$ be defined by

$$
C\left[\begin{array}{l}
\varphi \\
\psi
\end{array}\right]=\left.\frac{\partial \varphi}{\partial \nu}\right|_{\Gamma} \quad\left(\left[\begin{array}{l}
\varphi \\
\psi
\end{array}\right] \in \mathcal{D}(A)\right)
$$

It is well known that the $C$ is an admissible observation operator for the semigroup $\mathbb{T}$ generated by $A$.

Finally, for $\xi \in \Omega$ we define $f \in X_{-1}$ by

$$
f=\left[\begin{array}{c}
0 \\
\delta_{\xi}
\end{array}\right]
$$

By applying Proposition 3.2 with the above choice of spaces and operators we obtain

Proposition 4.1. Let $w_{0} \in H_{0}^{1}(\Omega)$ and $w_{1} \in H^{-1}(\Omega)$. Let $\Gamma$ be an open subset of $\partial \Omega$. Then, for any $\tau>0$ and $\lambda \in H^{1}(0, \tau), \lambda(0) \neq 0$, the system (1.7) admits a unique solution

$$
w \in C^{0}\left([0, \tau] ; H_{0}^{1}(\Omega)\right) \cap C^{1}\left([0, \tau] ; H^{-1}(\Omega)\right),
$$

such that $y$ defined by $(1.8)$ is in $L^{2}\left(0, \tau ; L^{2}(\Gamma)\right)$.

The main result of the section is the following

Theorem 4.2. Let $\tau>0$, let $\Omega \subset \mathbb{R}^{2}$ and let $\Gamma$ be a nonempty open subset of $\partial \Omega$ satisfying one of following conditions:

1. $\partial \Omega$ is smooth and $\Gamma$ satisfies the geometric optics conditions;

2. $\Omega$ is a rectangle and $\Gamma$ contains both a horizontal and a vertical segment of nonzero length. 
Let $\varepsilon>0$ and let $\xi^{(1)}, \xi^{(2)} \in \Omega$ be two points in $\Omega$, each one at distance at least $\varepsilon$ from $\partial \Omega$. Assume that $\lambda \in H^{1}(0, \tau)$ with $\lambda(0) \neq 0, w_{0} \in H_{0}^{1}(\Omega), w_{1} \in H^{-1}(\Omega)$ and denote

$$
y^{(j)}=\left.\frac{\partial w^{(j)}}{\partial \nu}\right|_{\Gamma} \quad j \in\{1,2\},
$$

where $w^{(j)}$ is the solution of $(1.7)$ with $\xi=\xi^{(j)}, j \in\{1,2\}$.

Then there exists $K>0$, depending only on $\Omega, \Gamma, \varepsilon$ and $\tau$ such that

$$
\left\|y^{(1)}-y^{(2)}\right\|_{L^{2}\left(0, \tau ; L^{2}(\Gamma)\right)} \geqslant K\left|\xi^{(1)}-\xi^{(2)}\right|,
$$

where $|\cdot|$ stands for the standard norm in $\mathbb{R}^{2}$.

Proof. We write

$$
z=\left[\begin{array}{c}
w \\
\frac{\partial w}{\partial t}
\end{array}\right], \quad f=\left[\begin{array}{c}
0 \\
\delta_{\xi^{(1)}}-\delta_{\xi^{(2)}}
\end{array}\right] .
$$

Then, with the above choice of spaces and operators, (1.7) and (1.8) can be written in the form (3.4), $(3.5)$.

On the other hand, applying Theorems 2.16 and 2.17 we know that the pair $(A, C)$ is exactly observable in any time $\tau>0$ if condition 1 (respectively condition 2) in the statement of the theorem is satisfied.

To apply Theorem 3.3, it remains to determine $Z$ and $Z^{\prime}$. We can take $\beta=0$ in (2.16) so that

$$
Z=A^{-1}\left(X+C^{*} Y\right) .
$$

To obtain the adjoint of $C$, we consider the operator $D \in \mathcal{L}\left(L^{2}(\Gamma) ; L^{2}(\Omega)\right)$ defined by

$$
\begin{cases}-\Delta(D g)=0 & \text { in } \Omega, \\ (D g)=0 & \text { on } \partial \Omega \backslash \Gamma, \\ (D g)=g & \text { on } \Gamma,\end{cases}
$$

for all $g \in L^{2}(\Gamma)$. In other words, $D g$ is the unique element of $L^{2}(\Omega)$ such that

$$
\int_{\Omega}(D g) \Delta \varphi \mathrm{d} x=\int_{\Gamma} g \frac{\partial \varphi}{\partial \nu} \mathrm{d} \sigma \quad\left(\varphi \in H^{2}(\Omega) \cap H_{0}^{1}(\Omega)\right) .
$$

We also define by $A_{0}$ the operator

$$
A_{0}: D\left(A_{0}\right)=H^{2}(\Omega) \cap H_{0}^{1}(\Omega) \rightarrow H=L^{2}(\Omega), \quad \varphi \mapsto-\Delta \varphi .
$$

The above operator is definite positive and invertible and we can consider its square root

$$
A_{0}^{1 / 2}: D\left(A_{0}^{1 / 2}\right)=H_{0}^{1}(\Omega) \rightarrow H,
$$

which is also invertible.

It is easy to check that the $H_{0}^{1}$ norm is equivalent to the norm

$$
\varphi \mapsto\left\|A_{0}^{1 / 2} \varphi\right\|_{L^{2}(\Omega)} .
$$

Let us consider $(\varphi, \psi) \in D(A)$ and $g \in L^{2}(\Gamma)=Y$. By definition of $C$, we have

$$
\left\langle C\left[\begin{array}{l}
\varphi \\
\psi
\end{array}\right], g\right\rangle_{L^{2}(\Gamma)}=\int_{\Gamma} g \frac{\partial \varphi}{\partial \nu} \mathrm{d} \sigma .
$$


Therefore,

$$
\left\langle C\left[\begin{array}{l}
\varphi \\
\psi
\end{array}\right], g\right\rangle_{L^{2}(\Gamma)}=\int_{\Omega}(D g) \Delta \varphi \mathrm{d} x=-\left\langle A_{0} \varphi, D g\right\rangle_{H} .
$$

Now, we notice that for $f \in D\left(A_{0}\right)$,

$$
\left\langle A_{0} \varphi, f\right\rangle_{H}=\langle\varphi, f\rangle_{D\left(A_{0}^{1 / 2}\right)}=\langle\varphi, f\rangle_{D\left(A_{0}^{3 / 2}\right), D\left(A_{0}^{-1 / 2}\right), D\left(A_{0}^{1 / 2}\right)}=\langle\varphi, f\rangle_{W, H^{-1}(\Omega), H_{0}^{1}(\Omega)}
$$

where $\langle\cdot, \cdot\rangle_{V, V^{\prime}, H}$ denotes the duality product of an element of $V$ and of an element of $V^{\prime}$, with respect to the pivot space $H$. By density, for $f \in H$ we have

$$
\left\langle A_{0} \varphi, f\right\rangle_{H}=\langle\varphi, f\rangle_{W, H^{-1}(\Omega), H_{0}^{1}(\Omega)}
$$

and thus

$$
\left\langle C\left[\begin{array}{l}
\varphi \\
\psi
\end{array}\right], g\right\rangle_{L^{2}(\Gamma)}=\left\langle\left[\begin{array}{l}
\varphi \\
\psi
\end{array}\right],\left[\begin{array}{c}
D g \\
0
\end{array}\right]\right\rangle_{D(A), D(A)^{*}} .
$$

We deduce from the above relation that for all $g \in L^{2}(\Gamma)$,

$$
C^{*} g=\left[\begin{array}{c}
D g \\
0
\end{array}\right] \text {. }
$$

This implies that

$$
Z=X_{1}+\{0\} \times D Y \subset W \times L^{2}(\Omega) .
$$

In particular, $H^{-1}(\Omega) \times W^{\prime} \subset Z^{\prime}$, with continuous imbedding.

Consequently Theorem 3.3 yields

$$
\left\|y^{(1)}-y^{(2)}\right\|_{L^{2}(\Gamma)} \geqslant \kappa_{\tau}\left\|\delta_{\xi^{(1)}}-\delta_{\xi^{(2)}}\right\|_{W^{\prime}}
$$

for some $\kappa_{\tau}>0$. This, together with

$$
\left\|\delta_{\xi^{(1)}}-\delta_{\xi^{(2)}}\right\|_{W^{\prime}} \geqslant \gamma\left|\xi^{(1)}-\xi^{(2)}\right|,
$$

imply the conclusion. The inequality (4.11) is proven in the Lemma 4.3.

The following result is very similar to results used and proved in $[11,12]$, but we cannot apply directly their results. However the proof of the next lemma is based on the same kind of arguments than in $[11,12]$.

Lemma 4.3. If $\varepsilon>0$ is small enough then there exists a positive constant $\gamma=\gamma(\Omega, \varepsilon)$ such that, for all $a, b \in \Omega$, with $\operatorname{dist}(a, \partial \Omega)>\varepsilon$ and $\operatorname{dist}(b, \partial \Omega)>\varepsilon$,

$$
|a-b| \leqslant \gamma\left\|\delta_{a}-\delta_{b}\right\|_{W^{\prime}} .
$$

Proof. We denote by $\Omega_{\varepsilon}$ the open set defined by

$$
\Omega_{\varepsilon}=\{x \in \Omega ; \operatorname{dist}(x, \partial \Omega)>\varepsilon\} .
$$

For $\varepsilon$ small enough, $\Omega_{\varepsilon}$ is not empty. There exists a function $\varphi_{1} \in W$ such that

$$
\varphi_{1}\left(x_{1}, x_{2}\right)=x_{1} \quad\left(\left(x_{1}, x_{2}\right) \in \Omega_{\varepsilon}\right) .
$$

Since $a, b \in \Omega_{\varepsilon}$, we have

$$
\left\langle\delta_{a}-\delta_{b}, \varphi_{1}\right\rangle=a_{1}-b_{1}
$$

Thus,

$$
\left|a_{1}-b_{1}\right| \leqslant \gamma_{1}(\Omega, \varepsilon)\left\|\delta_{a}-\delta_{b}\right\|_{W^{\prime}}
$$

We can use a similar argument for the second coordinate to conclude the proof of the lemma. 


\subsection{Recovery of sources in $H_{0}^{1}(\Omega)$}

We consider the initial value problem

$$
\begin{cases}\frac{\partial^{2} w}{\partial t^{2}}+\Delta^{2} w=\lambda(t) f & \text { in }(0, \tau) \times \Omega \\ w=\Delta w=0 & \text { on }(0, \tau) \times \partial \Omega \\ w(0, x)=w_{0}(x), \frac{\partial w}{\partial t}(0, x)=w_{1}(x) & x \in \Omega\end{cases}
$$

where $\lambda$ is given and satisfies $\lambda \in H^{1}(0, \tau)$ and $\lambda(0) \neq 0$. We aim to find $f \in H_{0}^{1}(\Omega)$ by knowing either

$$
\frac{\partial^{2} w}{\partial t \partial \nu} \text { or } \frac{\partial^{2} \Delta w}{\partial t \partial \nu}
$$

In this case, we obtain the following stability results.

Theorem 4.4. Let $\Omega, \Gamma, w_{0}, w_{1}, \tau$ and $\lambda$ satisfy the conditions of Theorem 4.2. Suppose that $w^{(j)}$ is the solution of (1.7) with $f=f^{(j)} \in H_{0}^{1}(\Omega), j \in\{1,2\}$. Then there exists $K>0$, depending only on $\Omega, \Gamma$ and $\tau$ such that

$$
\left\|\frac{\partial^{2} w^{(1)}}{\partial t \partial \nu}-\frac{\partial^{2} w^{(2)}}{\partial t \partial \nu}\right\|_{L^{2}\left(0, \tau ; L^{2}(\Gamma)\right)} \geqslant K\left\|f^{(1)}-f^{(2)}\right\|_{H_{0}^{1}(\Omega)}
$$

and

$$
\left\|\frac{\partial^{2} \Delta w^{(1)}}{\partial t \partial \nu}-\frac{\partial^{2} \Delta w^{(2)}}{\partial t \partial \nu}\right\|_{L^{2}\left(0, \tau ; L^{2}(\Gamma)\right)} \geqslant K\left\|f^{(1)}-f^{(2)}\right\|_{H_{0}^{1}(\Omega)} .
$$

Proof. We set

$$
\begin{gathered}
H=H_{0}^{1}(\Omega), \quad \mathcal{D}\left(A_{0}\right)=\left\{\varphi \in H^{5}(\Omega) \mid \varphi=\Delta \varphi=\Delta^{2} \varphi=0 \quad \text { on } \partial \Omega\right\}, \\
Y=L^{2}(\Gamma), \quad A_{0} \varphi=\Delta^{2} \varphi \quad \forall \varphi \in \mathcal{D}\left(A_{0}\right) .
\end{gathered}
$$

With this choice of spaces and operators, one can easily check that $A_{0}$ is self-adjoint, positive and boundedly invertible and that

$$
\mathcal{D}\left(A_{0}^{\frac{1}{2}}\right)=\left\{\varphi \in H^{3}(\Omega) \mid \varphi=\Delta \varphi=0 \quad \text { on } \partial \Omega\right\} .
$$

We set $X=\mathcal{D}\left(A_{0}^{\frac{1}{2}}\right) \times H, \mathcal{D}(A)=\mathcal{D}\left(A_{0}\right) \times \mathcal{D}\left(A_{0}^{\frac{1}{2}}\right)$ and

$$
A=\left[\begin{array}{cc}
0 & I \\
-A_{0} & 0
\end{array}\right]
$$

The observation operator is given by

$$
C\left[\begin{array}{l}
\varphi \\
\psi
\end{array}\right]=\left.\frac{\partial \psi}{\partial \nu}\right|_{\Gamma} \quad \forall\left[\begin{array}{l}
\varphi \\
\psi
\end{array}\right] \in \mathcal{D}\left(A_{0}\right) \times \mathcal{D}\left(A_{0}^{\frac{1}{2}}\right)
$$

and

$$
F=\left[\begin{array}{l}
0 \\
f
\end{array}\right] \in X .
$$


The system (4.12) can be written as (3.4)-(3.5) and from Theorems 2.16 and 2.17, the couple $(A, C)$ is exactly observable for all $\tau>0$. Using Theorem 3.3 , we deduce

$$
\left\|y^{(1)}-y^{(1)}\right\|_{H_{L}^{1}(0, \tau ; Y)} \geqslant \kappa_{\tau}\left\|f^{(1)}-f^{(2)}\right\|_{H_{0}^{1}(\Omega)} .
$$

To treat the other case, we consider the observation operator given by

$$
C\left[\begin{array}{l}
\varphi \\
\psi
\end{array}\right]=\left.\frac{\partial \Delta \varphi}{\partial \nu}\right|_{\Gamma} \quad \forall\left[\begin{array}{l}
\varphi \\
\psi
\end{array}\right] \in \mathcal{D}\left(A_{0}\right) \times \mathcal{D}\left(A_{0}^{\frac{1}{2}}\right)
$$

and we apply Corollary 2.18 to deduce that the couple $(A, C)$ is exactly observable for all $\tau>0$.

\subsection{Recovery of $H_{0}^{1}(\Omega)$ sources in the problem with potential}

We can also deduce some stability results for the inverse source problem for the plate equation with a potential $d \in H^{1+\alpha}(\Omega)$, with $\alpha>0$. Let us consider the system

$$
\begin{cases}\frac{\partial^{2} w}{\partial t^{2}}+\Delta^{2} w+d w=\lambda(t) f & \text { in }(0, \tau) \times \Omega \\ w=\Delta w=0 & \text { on }(0, \tau) \times \partial \Omega \\ w(0, x)=w_{0}(x), \frac{\partial w}{\partial t}(0, x)=w_{1}(x) & x \in \Omega\end{cases}
$$

where $\lambda$ is a given function satisfying $\lambda \in H^{1}(0, \tau)$ and $\lambda(0) \neq 0$. We aim to find $f \in H_{0}^{1}(\Omega)$ by knowing

$$
\frac{\partial \Delta w}{\partial \nu} \text { and } \frac{\partial w}{\partial \nu}
$$

Combining Theorems 3.3 and 2.19, we get the following result.

Theorem 4.5. Let $\tau>0, \lambda \in H^{1}(0, \tau), \lambda(0) \neq 0$ and $\Gamma$ an open subset of $\Omega$ satisfying one of the following assumptions:

1. $\partial \Omega$ is smooth and $\Gamma$ satisfies the geometric optics condition;

2. $\Omega$ is a rectangle and $\Gamma$ contains both a horizontal and a vertical segment of nonzero lengtht.

Let $f^{(1)}, f^{(2)} \in H_{0}^{1}(\Omega)$ and suppose that $w^{(j)}$ is the solution of $(4.13)$ with $f=f^{(j)}, j \in\{1,2\}$. Then there exists $K>0$, depending only on $\Omega, \Gamma$ and $\tau$ such that

$$
\left\|\frac{\partial \Delta w^{(1)}}{\partial \nu}-\frac{\partial \Delta w^{(2)}}{\partial \nu}\right\|_{L^{2}\left(0, \tau ; L^{2}(\Gamma)\right)}+\left\|\frac{\partial w^{(1)}}{\partial \nu}-\frac{\partial w^{(2)}}{\partial \nu}\right\|_{L^{2}\left(0, \tau ; L^{2}(\Gamma)\right)} \geqslant K\left\|f^{(1)}-f^{(2)}\right\|_{H_{0}^{1}(\Omega)} .
$$

\section{Inverse source problems for the plate equation with unknown in- tensities}

In this section, we establish an identifiability result for the unknown source term $\sum_{j=1}^{N} \lambda_{j}(t) \delta_{\xi_{j}}$ in the plates equation

$$
\begin{cases}\frac{\partial^{2} w}{\partial t^{2}}+\Delta^{2} w=\sum_{j=1}^{N} \lambda_{j}(t) \delta_{\xi_{j}} & \text { in }(0, \tau) \times \Omega, \\ w=\Delta w=0 & \text { on }(0, \tau) \times \partial \Omega \\ w(0, x)=w_{0}(x), \frac{\partial w}{\partial t}(0, x)=w_{1}(x) & \text { for } x \in \Omega\end{cases}
$$


where now the number $N$ of point sources, their locations $\xi_{j} \in \Omega$ and the functions $\lambda_{j}$ are all unknown. The problem involves now more unknowns and therefore we consider further boundary measurements than those used to solve the problem of Subsection 4.1. The method employed here is different from the method used in the previous section. This explains that the assumptions are quite different. In particular, we assume that $\lambda_{j}(0)=0$ and that there exists a time $\tau_{1} \in(0, \tau)$ such that

$$
\lambda_{j}(t)=0 \quad\left(t \geqslant \tau_{1}\right) .
$$

To deal with the corresponding inverse problem, we follow a method inspired by [8], based on the Fourier transformation.

We first present a regularity result for (5.14). In what follows, for $\varepsilon>0$, we set

$$
\Omega^{\varepsilon}=\{x \in \Omega \mid \operatorname{dist}(x, \partial \Omega)<\varepsilon\}
$$

and we use the notation from Subsection 4.1 for the spaces $X, W$ and the operator $A$, i.e.

$$
\begin{gathered}
X=H_{0}^{1}(\Omega) \times H^{-1}(\Omega), \quad W=\left\{\varphi \in H^{3}(\Omega) \mid \varphi=\Delta \varphi=0 \text { on } \partial \Omega\right\} \\
\mathcal{D}(A)=W \times H_{0}^{1}(\Omega) \\
A\left[\begin{array}{l}
\varphi \\
\psi
\end{array}\right]=\left[\begin{array}{c}
\psi \\
-\Delta^{2} \varphi
\end{array}\right] \quad\left(\left[\begin{array}{l}
\varphi \\
\psi
\end{array}\right] \in \mathcal{D}(A)\right) .
\end{gathered}
$$

We recall that, if we set $f_{j}=\left[\begin{array}{c}0 \\ \delta_{\xi_{j}}\end{array}\right], g(t)=\sum_{j=1}^{N} \lambda_{j}(t) f_{j}$ and $z_{0}=\left[\begin{array}{c}w_{0} \\ w_{1}\end{array}\right]$, the function $w$ is the solution of (5.14) if and only if $z=\left[\begin{array}{c}w \\ \frac{\partial w}{\partial t}\end{array}\right]$ is the solution of

$$
\begin{aligned}
& \dot{z}(t)=A z(t)+g(t) \quad(t \geqslant 0) \\
& z(0)=z_{0} .
\end{aligned}
$$

Henceforth, we will use also the notation $\mathcal{G}(t):=\sum_{j=1}^{N} \lambda_{j}(t) \delta_{\xi_{j}}$.

Proposition 5.1. Let

$$
\begin{gathered}
w_{0} \in H^{5}(\Omega), w_{0}=\Delta w_{0}=\Delta^{2} w_{0}=0 \text { on } \partial \Omega, \text { and } \\
w_{1} \in H^{3}(\Omega), w_{1}=\Delta w_{1}=0 \text { on } \partial \Omega .
\end{gathered}
$$

Let $\left\{\xi_{1}, \ldots, \xi_{N}\right\} \subset \Omega$ and $\varepsilon=\min _{j \in\{1, \ldots, N\}}\left\{\operatorname{dist}\left(\xi_{j}, \partial \Omega\right)\right\}$. Let $\Gamma$ be a nonempty open subset of $\partial \Omega$. Then, for any $\tau>0$ and $\lambda_{j} \in C^{2}([0, \tau])(j=0, \ldots, N)$ with $\lambda_{j}(0)=\dot{\lambda}_{j}(0)=0$, the system (5.14) admits a unique solution

$$
w \in C^{0}\left([0, \tau] ; H_{0}^{1}(\Omega)\right) \cap C^{0}\left([0, \tau] ; H^{5}\left(\Omega^{\varepsilon}\right)\right) \cap C^{1}\left([0, \tau] ; H^{3}\left(\Omega^{\varepsilon}\right)\right)
$$

such that

$$
\frac{\partial w}{\partial \nu} \in L^{2}\left(0, \tau ; L^{2}(\Gamma)\right), \quad \frac{\partial \Delta w}{\partial \nu} \in L^{2}\left(0, \tau ; L^{2}(\Gamma)\right)
$$

Proof. We consider the spaces and the operator defined by (5.16)-(5.17). A simple calculation shows that

$$
\mathcal{D}\left(A^{2}\right)=\left\{\varphi \in H^{5}(\Omega) \mid \varphi=\Delta \varphi=\Delta^{2} \varphi=0 \text { on } \partial \Omega\right\} \times\left\{\varphi \in H^{3}(\Omega) \mid \varphi=\Delta \varphi=0 \text { on } \partial \Omega\right\}
$$


and $z_{0}=\left[\begin{array}{l}w_{0} \\ w_{1}\end{array}\right] \in \mathcal{D}\left(A^{2}\right)$. Moreover, $g \in H^{2}\left(0, \tau, X_{-1}\right)$ (recall that $\left.X_{-1}=H^{-1}(\Omega) \times W^{\prime}\right)$. Then $z=\left[\begin{array}{c}w \\ \frac{\partial w}{\partial t}\end{array}\right] \in C^{0}([0, \tau] ; X)$. The additional assumptions on the data will allow to improve this result.

Since the problem is linear we can analyse separately the problem (5.14) with $\mathcal{G} \equiv 0$ and the problem (5.14) with $w_{0} \equiv w_{1} \equiv 0$.

In the first case, we get from classical theory on semigroups that $z=\left[\begin{array}{c}w \\ \frac{\partial w}{\partial t}\end{array}\right] \in C^{0}\left([0, \tau] ; \mathcal{D}\left(A^{2}\right)\right)$ and therefore $\frac{\partial w}{\partial \nu}$ and $\frac{\partial \Delta w}{\partial \nu}$ have the stated summability properties. In the second case, we note that each $\lambda_{j}$ can be written in the form

$$
\lambda_{j}(t)=\int_{0}^{t}\left(\int_{0}^{s} \ddot{\lambda}_{j}(\theta) \mathrm{d} \theta\right) \mathrm{d} s,
$$

and that the unique solution of (5.14) is given by

$$
w(t, x)=\int_{0}^{t}\left(\int_{0}^{s} u(\theta, x) \mathrm{d} \theta\right) \mathrm{d} s,
$$

where $u \in C^{0}\left([0, \tau] ; H_{0}^{1}(\Omega)\right) \cap C^{1}\left([0, \tau] ; H^{-1}(\Omega)\right)$ solves $(5.14)$ when $w_{0} \equiv w_{1} \equiv 0$ and the source term is $\mathcal{G}(t)=\sum_{j=1}^{N} \ddot{\lambda}_{j}(t) f_{j}$. Therefore, $w \in C^{2}\left([0, \tau] ; H^{1}(\Omega)\right)$ and we deduce that

$$
\frac{\partial^{2} w}{\partial t^{2}} \in C^{0}\left([0, \tau] ; H^{1}(\Omega)\right)
$$

From equation (5.14) and classical regularity results for the elliptic problem associated with the bilaplacian, we obtain

$$
w \in C^{0}\left([0, \tau] ; H^{5}\left(\Omega^{\varepsilon}\right)\right),
$$

which implies the desired trace properties.

Based on Proposition 5.1 we will consider measurements of $\frac{\partial w}{\partial \nu}$ and $\frac{\partial \Delta w}{\partial \nu}$ on parts of $\partial \Omega$ until time $\tau_{1}$ and continue the measurement of $\frac{\partial w}{\partial \nu}$ until time $\tau$. In accordance with the operator formulation (5.18) with (5.16)-(5.17) and $z_{0} \in \mathcal{D}\left(A^{2}\right)$, we introduce the following observation operators for the inverse source problem for (5.14). The output spaces are $Y_{1}=L^{2}(\Gamma) \times L^{2}\left(\Gamma_{1}\right)$ and $Y_{2}=L^{2}(\Gamma)$, and the operators $C_{1} \in \mathcal{L}\left(V_{1}, Y_{1}\right)$, with

$$
V_{1}=\left\{u \in H_{0}^{1}(\Omega)|u|_{\Omega^{\varepsilon}} \in H^{5}\left(\Omega^{\varepsilon}\right)\right\}
$$

and $C_{2} \in \mathcal{L}\left(X_{1}, Y_{2}\right)$ are defined by

$$
\begin{gathered}
C_{1}\left[\begin{array}{l}
\varphi \\
\psi
\end{array}\right]=\left[\begin{array}{c}
\left.\frac{\partial \varphi}{\partial \nu}\right|_{\Gamma} \\
\left.\frac{\partial \Delta \varphi}{\partial \nu}\right|_{\Gamma_{1}}
\end{array}\right] \quad\left(\left[\begin{array}{l}
\varphi \\
\psi
\end{array}\right] \in V_{1}\right), \\
C_{2}\left[\begin{array}{c}
\varphi \\
\psi
\end{array}\right]=\left.\frac{\partial \varphi}{\partial \nu}\right|_{\Gamma} \quad\left(\left[\begin{array}{c}
\varphi \\
\psi
\end{array}\right] \in \mathcal{D}(A)\right) .
\end{gathered}
$$

Hence, the output function corresponding to the measured data in this case is given by

$$
y(t)= \begin{cases}C_{1} z(t), & \left(t \in\left[0, \tau_{1}\right]\right), \\ C_{2} z(t), & \left(t \in\left[\tau_{1}, \tau\right]\right) .\end{cases}
$$

The main result of this section is the following 
Theorem 5.2. Consider the sources $\mathcal{G}^{(l)}=\sum_{j=1}^{N^{(l)}} \lambda_{j}^{(l)} \delta_{\xi_{j}^{(l)}}, l \in\{1,2\}$, in the plate equation (5.14) and assume that $\lambda_{j}^{(l)} \in C^{2}([0, \tau]), j \in\{1, \ldots, N\}$ and $l \in\{1,2\}$, satisfy $\lambda_{j}^{(l)}(0)=\dot{\lambda}_{j}^{(l)}(0)=0$ and $\lambda_{j}^{(l)}(t)=0$, for $t \geqslant \tau_{1}$. Let $w^{(1)}$ and $w^{(2)}$ be the corresponding solutions of (5.14) with initial condition

$$
\begin{gathered}
w_{0} \in H^{5}(\Omega), w_{0}=\Delta w_{0}=\Delta^{2} w_{0}=0 \text { on } \partial \Omega, \text { and } \\
w_{1} \in H^{3}(\Omega), w_{1}=\Delta w_{1}=0 \text { on } \partial \Omega .
\end{gathered}
$$

Let $\Gamma, \Gamma_{1}$ be two nonempty open subset of $\partial \Omega$ with $\Gamma \subseteq \Gamma_{1}$ and assume that one of the following assumptions hold:

1. $\partial \Omega$ is smooth and $\Gamma$ satisfies the geometric optics conditions;

2. $\Omega$ is a rectangle and $\Gamma$ contains both a horizontal and a vertical segment of nonzero length.

If

$$
\frac{\partial w^{(1)}}{\partial \nu}=\frac{\partial w^{(2)}}{\partial \nu} \text { on }(0, \tau) \times \Gamma, \quad \frac{\partial \Delta w^{(1)}}{\partial \nu}=\frac{\partial \Delta w^{(2)}}{\partial \nu} \text { on }\left(0, \tau_{1}\right) \times \Gamma_{1}
$$

then $\mathcal{G}^{(1)}=\mathcal{G}^{(2)}$.

Proof. We denote by $z^{(1)}, z^{(2)}$ the solutions of (5.18) for $g^{(1)}(t)=\sum_{j=1}^{N^{(1)}} \lambda_{j}^{(1)}(t) f_{j}^{(1)}$ and $g^{(2)}(t)=$ $\sum_{j=1}^{N^{(2)}} \lambda_{j}^{(2)}(t) f_{j}^{(2)}$, respectively, and by $y^{(1)}, y^{(2)}$ the corresponding observations given by (5.21)-(5.22). We assume that $y^{(1)}(t)=y^{(2)}(t)$, for $t \in(0, \tau)$. Let us write $z(t):=z^{(1)}(t)-z^{(2)}(t)$ and $y(t):=$ $y^{(1)}(t)-y^{(2)}(t)$. Since $g^{(l)}(t)=0$, for $t \geqslant \tau_{1},(l \in\{1,2\})$ the functions $z$ and $y$ satisfy

$$
\begin{aligned}
& \dot{z}(t)=A z(t) \quad\left(t \in\left(\tau_{1}, \tau\right)\right), \\
& z\left(\tau_{1}\right) \in X, \\
& y(t)=0 \quad\left(t \in\left(\tau_{1}, \tau\right)\right) .
\end{aligned}
$$

We notice that $y(t)=\left[\Psi_{\tau-\tau_{1}} z\left(\tau_{1}\right)\right]\left(t-\tau_{1}\right)$. We set $\tau_{0}=\tau-\tau_{1}>0$. Since the pair $\left(A, C_{2}\right)$, with $A$ defined by (5.17) and $C_{2}$ defined by (5.22), is exactly observable in time $\tau_{0}>0$, we have

$$
\|y\|_{L^{2}\left(\tau_{1}, \tau ; Y\right)} \geqslant \kappa_{\tau}\left\|z\left(\tau_{1}\right)\right\|_{X},
$$

and therefore $z\left(\tau_{1}\right)=0$. Thus, $z$ satisfies

$$
\begin{aligned}
& \dot{z}(t)=A z(t)+g^{(1)}-g^{(2)} \quad\left(t \in\left(0, \tau_{1}\right)\right) \\
& z(0)=0, \quad z\left(\tau_{1}\right)=0 .
\end{aligned}
$$

Extending $\lambda_{j}^{(l)}, z$ and $y$ by zero outside $\left(0, \tau_{1}\right)$, and then applying Fourier transformation in the variable $t$ yield

$$
(i \varpi I-A) \widehat{z}(\varpi)=\sum_{j=1}^{N^{(1)}} \widehat{\lambda_{j}^{(1)}}(\varpi) f_{j}^{(1)}-\sum_{j=1}^{N^{(2)}} \widehat{\lambda_{j}^{(2)}}(\varpi) f_{j}^{(2)}, \quad \forall \varpi \in \mathbb{R} .
$$

where the notation $\widehat{\cdot}$ indicates the Fourier transform of the extended function. Setting $\widehat{z}(\varpi)=\left[\begin{array}{l}v \\ \vartheta\end{array}\right]$, we conclude that $v$ satisfies

$$
\Delta^{2} v-\varpi^{2} v=\widehat{\mathcal{G}} \text { in } \Omega
$$

where we have set $\mathcal{G}=\mathcal{G}^{(1)}-\mathcal{G}^{(2)}$. 
Since $\widehat{\mathcal{G}} \in \mathcal{E}^{\prime}\left(\mathbb{R}^{2}\right)$ (distribution with compact support) with support contained in $\Omega$ we can extend the left hand side of (5.23) by zero outside $\Omega$ and get

$$
\widetilde{\Delta^{2} v}-\varpi^{2} \widetilde{v}=\widehat{\mathcal{G}} \text { in } \mathbb{R}^{2}
$$

where the notation $\sim$ indicates the extension to $\mathbb{R}^{2}$ with respect to the variable $x$. The next lemma gives a relation between $\widetilde{\Delta^{2} v}$ and $\Delta^{2} \widetilde{v}$. This result is an easy extension of a well-known result (see for instance Theorem 5.4.13 in [4]) so we omit the proof.

Lemma 5.3. Let $v \in V_{1}$ be such that $\Delta v=0$ on $\partial \Omega$. Let $\widetilde{v}$ and $\widetilde{\Delta^{2} v}$ be the extensions by zero outside $\Omega$ of $v$ and $\Delta^{2} v$, respectively. Then

$$
\widetilde{\Delta^{2} v}=\Delta^{2} \widetilde{v}+\frac{\partial \Delta v}{\partial \nu} \delta_{\partial \Omega}+\Delta\left(\frac{\partial v}{\partial \nu} \delta_{\partial \Omega}\right) \text { in } \mathcal{D}^{\prime}\left(\mathbb{R}^{2}\right)
$$

In the above lemma, we have used the notation

$$
\left\langle\delta_{\partial \Omega}, \varphi\right\rangle_{\mathcal{D}^{\prime}\left(\mathbb{R}^{2}\right), \mathcal{D}\left(\mathbb{R}^{2}\right)}=\int_{\partial \Omega} \varphi \mathrm{d} \Gamma .
$$

Now we go back to (5.24) and use Lemma 5.3 to conclude that $\widetilde{v}$ satisfies the following relation in the distributional sense

$$
\Delta^{2} \widetilde{v}-\varpi^{2} \widetilde{v}=\widehat{\mathcal{G}}+\frac{\partial \Delta v}{\partial \nu} \delta_{\partial \Omega}+\Delta\left(\frac{\partial v}{\partial \nu} \delta_{\partial \Omega}\right) \text { in } \mathbb{R}^{2},(\varpi \in \mathbb{R}) .
$$

Let

$$
\Phi_{0}(x)=\frac{1}{8 \pi}|x|^{2} \ln (|x|) \quad\left(x \in \mathbb{R}^{2} \backslash\{0\}\right)
$$

and for each $\varpi \in \mathbb{R} \backslash\{0\}$, let

$$
\Phi_{\varpi}(x)=\frac{i}{8|\varpi|}\left(H_{0}^{(1)}(\sqrt{|\varpi|}|x|)-H_{0}^{(1)}(i \sqrt{|\varpi|}|x|)\right) \quad\left(x \in \mathbb{R}^{2} \backslash\{0\}\right),
$$

where $H_{1}^{(0)}$ denotes the the first kind Hänkel function of order 0 . Then, as shown in Kitahara [10, pg.211], we have

$$
\left(\Delta^{2}-\varpi^{2}\right) \Phi_{\varpi}=\delta_{0} \text { in } \mathbb{R}^{2}
$$

i.e. $\Phi_{\varpi}$ is a fundamental solution of $\Delta^{2}-\varpi^{2} I$ in $\mathbb{R}^{2}$. We recall (see, for instance, Abramowitz and Stegun [1, pg.358]) that

$$
H_{0}^{(1)}(y)=J_{0}(y)+i Y_{0}(y) \quad(y \in \mathbb{R}),
$$

where $J_{0}$ (Bessel function of the first kind and of order zero) and $Y_{0}$ (Bessel function of the second kind and of order zero). The function $J_{0}$ is analytic in $\mathbb{R}$ with series expansion

$$
J_{0}(y)=\sum_{k=0}^{\infty} \frac{(-1)^{k} y^{2 k}}{2^{2 k}(k !)^{2}} \quad(y \in \mathbb{R})
$$

whereas $Y_{0}(y)$ can be represented as

$$
Y_{0}(y)=\frac{2}{\pi}\left(\ln \left(\frac{y}{2}\right)+\gamma\right) J_{0}(y)+\frac{2}{\pi} \sum_{k=1}^{\infty} \frac{(-1)^{k+1}}{(k !)^{2}}\left(\sum_{m=1}^{k} \frac{1}{m}\right)\left(\frac{y^{2}}{2}\right)^{k} \quad(y \in \mathbb{R} \backslash\{0\}),
$$


with $\gamma$ the Euler-Mascheroni constant; see [1, pg.360]. Therefore each $\Phi_{\varpi}$ is analytic in $\mathbb{R}^{2} \backslash\{0\}$.

We note that

$$
\mathcal{G}:=\sum_{j=1}^{N^{(1)}} \lambda_{j}^{(1)} \delta_{\xi_{j}^{(1)}}-\sum_{j=1}^{N^{(2)}} \lambda_{j}^{(2)} \delta_{\xi_{j}^{(2)}}
$$

can be written

$$
\mathcal{G}=\sum_{j=1}^{N} \mu_{j} \delta_{\xi_{j}}, \quad \text { so that } \quad \widehat{\mathcal{G}}=\sum_{j=1}^{N} \widehat{\mu_{j}} \delta_{\xi_{j}},
$$

by taking $N=\max \left\{N^{(1)}, N^{(2)}\right\}$.

Using the fundamental solutions (5.27)-(5.26) and the relation (5.25), we can write $\widetilde{v}$ as

$$
\widetilde{v}=\Phi_{\varpi} *\left(\widehat{\mathcal{G}}+\frac{\partial \Delta v}{\partial \nu} \delta_{\partial \Omega}+\Delta\left(\frac{\partial v}{\partial \nu} \delta_{\partial \Omega}\right)\right)
$$

from which we obtain the representation

$$
\widetilde{v}(x)=\sum_{j=1}^{N} \widehat{\mu_{j}} \Phi_{\varpi}\left(x-\xi_{j}\right)+\int_{\partial \Omega} \frac{\partial v}{\partial \nu}(y) \Delta \Phi_{\varpi}(x-y) \mathrm{d} \sigma_{y}+\int_{\partial \Omega} \frac{\partial \Delta v}{\partial \nu}(y) \Phi_{\varpi}(x-y) \mathrm{d} \sigma_{y} .
$$

Since

$$
\frac{\partial w^{(1)}}{\partial \nu}=\frac{\partial w^{(2)}}{\partial \nu} \text { on }(0, \tau) \times \Gamma, \quad \frac{\partial \Delta w^{(1)}}{\partial \nu}=\frac{\partial \Delta w^{(2)}}{\partial \nu} \text { on }\left(0, \tau_{1}\right) \times \Gamma_{1}
$$

it follows that

$$
\frac{\partial v}{\partial \nu}=\frac{\partial \Delta v}{\partial \nu}=0 \text { on } \Gamma_{1}
$$

which, in turn, implies that

$$
\widetilde{v}(x)=\sum_{j=1}^{N} \widehat{\mu_{j}} \Phi_{\varpi}\left(x-\xi_{j}\right)+\int_{\partial \Omega \backslash \Gamma_{1}} \frac{\partial v}{\partial \nu}(y) \Delta \Phi_{\varpi}(x-y) \mathrm{d} \sigma_{y}+\int_{\partial \Omega \backslash \Gamma_{1}} \frac{\partial \Delta v}{\partial \nu}(y) \Phi_{\varpi}(x-y) \mathrm{d} \sigma_{y} .
$$

Since $\Phi_{\varpi}$ is analytic in $\mathbb{R}^{2} \backslash\{0\},(5.31)$ shows that $\widetilde{v}$ is analytic in the connected domain

$$
\mathcal{W}:=\left[\mathbb{R}^{2} \backslash\left(\left\{\xi_{1}, \cdots, \xi_{N}\right\} \cup \partial \Omega\right)\right] \cup \Gamma_{1} .
$$

From the fact that $\widetilde{v}$ vanishes outside $\Omega$, it follows that $\widetilde{v} \equiv 0$ in $\mathcal{W}$.

Our aim now is to show that $\widehat{\mu_{j}} \equiv 0$. Based on the expansions (5.28) and (5.29), we can show that 
each function $\Phi_{\varpi}(x)(\varpi \neq 0)$ satisfies

$$
\begin{aligned}
\Phi_{\varpi}(x)= & \frac{i}{8|\varpi|}\left(H_{0}^{(1)}(\sqrt{|\varpi|}|x|)-H_{0}^{(1)}(i \sqrt{|\varpi|}|x|)\right) \\
= & \frac{-1}{4 \pi|\varpi|}(\ln (\sqrt{|\varpi|}|x| / 2)-\ln (i \sqrt{|\varpi|}|x| / 2))+O(|x|) \quad(|x| \rightarrow 0) \\
= & \frac{i}{8|\varpi|}+O(|x|) \quad(|x| \rightarrow 0) \\
\nabla \Phi_{\varpi}(x)= & \frac{i}{8 \sqrt{|\varpi|}} \frac{x}{|x|}\left(\dot{H}_{0}^{(1)}(\sqrt{|\varpi|}|x|)-i \dot{H}_{0}^{(1)}(i \sqrt{|\varpi|}|x|)\right) \\
= & x\left(\frac{2 \gamma-3}{8 \pi}-\frac{i}{16}+\frac{1}{4 \pi} \ln (\sqrt{|\varpi|}|x| / 2)+O(|x|)\right) \quad(|x| \rightarrow 0), \\
\Delta \Phi_{\varpi}(x)= & \frac{i}{8 \sqrt{|\varpi|}|x|}\left(\dot{H}_{0}^{(1)}(\sqrt{|\varpi|}|x|)-i \dot{H}_{0}^{(1)}(i \sqrt{|\varpi|}|x|)\right) \\
& +\frac{i}{8}\left(\ddot{H}_{0}^{(1)}(\sqrt{|\varpi|}|x|)+\ddot{H}_{0}^{(1)}(i \sqrt{|\varpi|}|x|)\right) \\
= & \frac{\gamma-1}{2 \pi}-\frac{i}{8}+\frac{1}{2 \pi} \ln (\sqrt{|\varpi|}|x| / 2)+O(|x|) \quad(|x| \rightarrow 0),
\end{aligned}
$$

and therefore

$$
\begin{aligned}
& \lim _{x \rightarrow \xi_{j}} \Phi_{\varpi}\left(x-\xi_{j}\right)=\frac{i}{8|\varpi|}, \\
& \lim _{x \rightarrow \xi_{j}} \nabla \Phi_{\varpi}\left(x-\xi_{j}\right)=0, \\
& \lim _{x \rightarrow \xi_{j}}\left|\Delta \Phi_{\varpi}\left(x-\xi_{j}\right)\right|=\infty,
\end{aligned}
$$

when $\varpi \neq 0$. Moreover,

$$
\begin{aligned}
\lim _{x \rightarrow \xi_{j}} \Phi_{0}\left(x-\xi_{j}\right) & =0, \\
\lim _{x \rightarrow \xi_{j}} \nabla \Phi_{0}\left(x-\xi_{j}\right) & =0, \\
\lim _{x \rightarrow \xi_{j}}\left|\Delta \Phi_{0}\left(x-\xi_{j}\right)\right| & =\infty .
\end{aligned}
$$

Now, we multiply $\Delta \widetilde{v}$ by $\frac{1}{\Delta \Phi_{\varpi}\left(x-\xi_{j}\right)}$ and let $x \rightarrow \xi_{j}$, which, from (5.31) yields

$$
\widehat{\mu_{j}}=\lim _{x \rightarrow \xi_{j}} \frac{\Delta \widetilde{v}(x)}{\Delta \Phi_{\varpi}\left(x-\xi_{j}\right)}=0
$$

for all $j \in\{1, \ldots, N\}$, since

$$
\lim _{x \rightarrow \xi_{j}} \frac{1}{\Delta \Phi_{\varpi}\left(x-\xi_{j}\right)}=0
$$

and since

$$
\lim _{x \rightarrow \xi_{j}} \Delta\left(\int_{\partial \Omega \backslash \Gamma_{1}} \frac{\partial v}{\partial \nu}(y) \Delta \Phi_{\varpi}(x-y) \mathrm{d} \sigma_{y}+\int_{\partial \Omega \backslash \Gamma_{1}} \frac{\partial \Delta v}{\partial \nu}(y) \Phi_{\varpi}(x-y) \mathrm{d} \sigma_{y}\right)
$$

is finite.

Applying the inverse Fourier transformation, we deduce $\mu_{j}=0$ for all $j \in\{1, \ldots, N\}$, and conclude that $\mathcal{G}^{(1)}=\mathcal{G}^{(2)}$. 


\section{References}

[1] M. Abramowitz And I. A. Stegun, Handbook of mathematical functions with formulas, graphs, and mathematical tables, Reprint of the 1972 edition, Dover Publications, Inc., New York, 1992.

[2] C. J. S. Alves And A. L. Silvestre, On the determination of point-forces on a stokes system, Math. Comput. Simulation, 66 (2004), pp. 385-397.

[3] C. Bardos, G. Lebeau, And J. Rauch, Sharp sufficient conditions for the observation, control, and stabilization of waves from the boundary, SIAM J. Control Optim., 30 (1992), pp. 1024-1065.

[4] J.-M. Bony, Cours d'analyse. Thorie des distributions et analyse de Fourier, Les Éditions de l'École Polytechnique, Palaiseau, 2001.

[5] S. Dolecki And D. L. Russell, A general theory of observation and control., SIAM J. Control Optimization, 15 (1977), pp. 185-220.

[6] R. G. Douglas, On majorization, factorization, and range inclusion of operators on Hilbert space, Proc. Amer. Math. Soc., 17 (1966), pp. 413-415.

[7] A. El Badia And T. Ha-Duong, Determination of point wave sources by boundary measurements, Inverse Problems, 17 (2001), pp. 1127-1139. Special issue to celebrate Pierre Sabatier's 65th birthday (Montpellier, 2000).

[8] — On an inverse source problem for the heat equation. Application to a pollution detection problem, J. Inverse Ill-Posed Probl., 10 (2002), pp. 585-599.

[9] M. L. J. Hautus, Controllability and observability conditions of linear autonomous systems, Nederl. Akad. Wetensch. Proc. Ser. A 72 = Indag. Math., 31 (1969), pp. 443-448.

[10] M. Kitahara, Boundary integral equation methods in eigenvalue problems of elastodynamics and thin plates, Studies in Applied Mechanics, 10, Elsevier Scientific Publishing Co., Amsterdam, 1985.

[11] V. Komornik And M. Yamamoto, Upper and lower estimates in determining point sources in a wave equation, Inverse Problems, 18 (2002), pp. 319-329.

[12] _ Estimation of point sources and applications to inverse problems, Inverse Problems, 21 (2005), pp. 2051-2070.

[13] R. KRess, Linear integral equations, vol. 82 of Applied Mathematical Sciences, Springer-Verlag, Berlin, 1989.

[14] G. Lebeau, Contrôle de l'équation de Schrödinger, J. Math. Pures Appl. (9), 71 (1992), pp. 267291.

[15] S. Nicaise AND O. ZAÏR, Identifiability, stability and reconstruction results of point sources by boundary measurements in heteregeneous trees, Rev. Mat. Complut., 16 (2003), pp. 151-178.

[16] — Determination of point sources in vibrating beams by boundary measurements: identifiability, stability, and reconstruction results, Electron. J. Differential Equations, (2004), pp. No. 20, 17 pp. (electronic). 
[17] A. PAZY, Semigroups of linear operators and applications to partial differential equations, vol. 44 of Applied Mathematical Sciences, Springer-Verlag, New York, 1983.

[18] J.-P. Puel and M. Yamamoto, Applications de la contrôlabilité exacte à quelques problèmes inverses hyperboliques, C. R. Acad. Sci. Paris Sér. I Math., 320 (1995), pp. 1171-1176.

[19] D. Salamon, Infinite-dimensional linear systems with unbounded control and observation: a functional analytical approach, Trans. Amer. Math. Soc., 300 (1987), pp. 383-431.

[20] — Realization theory in Hilbert space, Mathematical Systems Theory, 21 (1989), pp. 147-164.

[21] G. Tenenbaum and M. Tucsnak, Fast and strongly localized observation for the Schrödinger equation, Transactions of the American Mathematical Society, (2007), p. à paraître.

[22] M. TuCsnak And G. WEIss, Simultaneous exact controllability and some applications, SIAM J. Control Optim., 38 (2000), pp. 1408-1427 (electronic).

[23] M. TuCSNAK AND G. WeIss, Observation and control for operator semigroups, 2008. submitted book.

[24] Y. H. WANG, Global uniqueness and stability for an inverse plate problem, J. Optim. Theory Appl., 132 (2007), pp. 161-173.

[25] G. WeIss, Regular linear systems with feedback, Mathematics of Control, Signals and Systems, 7 (1994), pp. 23-57.

[26] - Transfer functions of regular linear systems, Part I: Characterizations of regularity, Trans. Amer. Math. Society, 342 (1994), pp. 827-854.

[27] M. Yамамото, Stability, reconstruction formula and regularization for an inverse source hyperbolic problem by a control method, Inverse Problems, 11 (1995), pp. 481-496.

[28] — Uniqueness and stability in multidimensional hyperbolic inverse problems, J. Math. Pures Appl. (9), 78 (1999), pp. 65-98. 